\title{
Code of Practice for Quality Assurance of Brachytherapy with Ir-192 Afterloaders
}

\author{
NEDERLANDSE COMMISSIE VOOR STRALINGSDOSIMETRIE
}

Report $\mathbf{3 0}$ of the Netherlands Commission on Radiation Dosimetry 


\section{Disclaimer regarding NCS reports}

The NCS frequently publishes reports for fellow professionals in which recommendations are given for various quality control procedures or otherwise. The members of the NCS board and the members of the concerning subcommittee do not claim any authority exceeding that of their professional expertise. Responsibility on how the NCS recommendations are implemented lies with the user, taking into account the practice in his/her institution. 


\section{Acknowledgements}

The NCS subcommittee wants to express her gratitude to the NCS-board and the following persons for their comments on and contributions to (parts) of this report:

Anton Mans, Medical Physics Expert

Celine van Beveren, Medical Physics Expert in training

Hetty vd Berg, Radiation Oncologist

Jack Venselaar, Medical Physics Expert

Lau Mestrom, Medical Physics Engineer

Lia Verhoef, Radiation Oncologist

Rita Reymen, Medical Physics Expert 


\section{Preface}

The Nederlandse Commissie voor Stralingsdosimetrie (NCS, Netherlands Commission on Radiation Dosimetry, http://www.radiationdosimetry.org) was officially established on 3rd September, 1982 with the aim of promoting the appropriate use of dosimetry of ionising radiation both for scientific research and for practical applications. The NCS is chaired by a board of scientists, made up via recommendations from the supporting societies, including the Nederlandse Vereniging voor Radiotherapie en Oncologie (Dutch Society for Radiotherapy and Oncology), the Nederlandse Vereniging voor Nucleaire Geneeskunde (Dutch Society of Nuclear Medicine), the Nederlandse Vereniging voor Klinische Fysica (Society for Medical Physics of the Netherlands), the Nederlandse Vereniging voor Radiobiologie (Netherlands Radiobiological Society), the Nederlandse Vereniging voor Stralingshygiëne (Netherlands Society for Radiological Protection), the Nederlandse Vereniging voor Medische Beeldvorming en Radiotherapie (Dutch Society for Medical Imaging and Radiotherapy), the Nederlandse Vereniging van Klinisch Fysisch Medewerkers (Dutch Society for Medical Physics Engineers), the Nederlandse Vereniging voor Radiologie (Radiological Society of the Netherlands) and the Belgische Vereniging voor Ziekenhuisfysici/Société Belge des Physiciens des Hôpitaux (Belgian Hospital Physicists Association) and expanded with a representative from the Dutch Metrology Institute VSL. To achieve its aims, the NCS carries out the following tasks: participation in dosimetry standardisation, promotion of mutual comparisons of dosimetry, drafting of dosimetry protocols and the collection and evaluation of physical data related to dosimetry. Furthermore, the commission shall establish or maintain links with national and international organisations concerned with ionising radiation and promulgate information on new developments in the field of radiation dosimetry.

Current members of the board of the NCS

J.B. van de Kamer, Chairman

T.W.M. Grimbergen, Vice-Chairman

J.A. de Pooter, Secretary

J.M.J. Hermans, Treasurer

A. Rijnders

N. De Graaf

F.W. Wittkämper

M.K. de Fluiter-Zeeman

J.R. de Jong

P. Sminia

N.A.P. Franken 


\section{Code of Practice for Quality Assurance of Brachytherapy with Ir-192 Afterloaders}

May 2018
This report was prepared by a subcommittee of the Netherlands Commission on Radiation
Dosimetry (NCS), consisting of Belgian and Dutch scientists.
Members of the subcommittee:
J. Steenhuijsen, Chairman
M. Harbers
A. Hoffmann
A. de Leeuw
A. Rijnders
M. Unipan

NCS, Delft, The Netherlands

For more information on NCS reports, see http://www.radiationdosimetry.org 


\section{Summary}

The Netherlands Commission on Radiation Dosimetry (NCS) has published 3 reports on brachytherapy with Ir-192 [1-3]. The goal of the current report is to update and partially replace these three previous reports of the NCS. This resulted in a code of practice for Quality Assurance for Ir-192 Brachytherapy.

The scope of the report has evolved from source strength determination to quality assurance of the whole brachytherapy delivery process, defined as:

A control computer sends a calibrated source from an afterloader through a transfer tube to an intended location in an applicator for an intended period of time, in a controlled manner and safe for patient and employees.

Chapters 2 through 5 describe the Quality Assurance steps and procedures to be performed on the equipment (afterloader, source and treatment room), starting with a description of QA of the source in Chapter 2. Chapter 3 treats the quality assurance on applicators and transfer tubes, which was previously rarely mentioned. Chapter 4 deals with radiation safety as well as security aspects regarding high-activity sealed sources. Chapter 5 concerns the specific parts of the Dutch "Convenant Veilige Toepassing Medische Technologie" with regard to brachytherapy. Although this "Convenant" specifically applies to the Dutch situation, ideas behind it to guarantee safe application of medical technology are valid worldwide. Of special significance is chapter 6 concerning the organizational aspects of the brachytherapy delivery process. Working together as a team, with clear responsibilities and tasks, will increase the safety of the procedure for patients and employees.

The subcommittee had its first meeting on 10 March 2014. 


\section{Abbreviations}

AAPM

$A K R$

AKS

ASTRO

CBRN

CE

CEWAZ

CT

CTB

EBT

ESTRO

EU

FANC

FCO

GEC-ESTRO

HASS

HDR

IAEA

ICRU

IEC

Ir-192

ISO

LDR

MPE

MR

NCS

NFU

NVZ

PDR

QA

QC

QUATRO

RAKR

RIAS
American Association of Physicists in Medicine

Air Kerma Rate

Air Kerma Strength

American Society for Radiation Oncology

Chemical, Biological, Radiological and Nuclear

Conformité Européenne (European Conformity)

Academic Hospitals Workgroup 'Medical devices without CE-marking'

Computed Tomography

Customer Technical Bulletin

External Beam Therapy

European Society for Radiotherapy and Oncology

European Union

Federaal Agentschap voor Nucleaire Controle - Belgian Federal Agency for Nuclear Control

Field Change Order

Groupe Européen de Curiethérapie- European Society for Radiotherapy and Oncology

High-activity sealed source

High Dose Rate

International Atomic Energy Agency

International Commission on Radiation Units and Measurements

International Electrotechnical Commission

Iridium-192 is a radioactive isotope of Iridium with a half-life of 73.83 days, used in brachytherapy as a radiation source

International Organization for Standardization

Low Dose Rate

Medical Physics Expert

Magnetic Resonance

Nederlandse Commissie voor Stralingsdosimetrie (Netherlands Commission on Radiation Dosimetry)

Netherlands Federation of University Medical Centers

Nederlandse Vereniging van Ziekenhuizen (The Dutch Hospital Association)

Pulsed Dose Rate

Quality Assurance (set of policies and procedures to maintain the quality of patient care)

Quality Control (set of procedures/tests to validate equipment is operating within tolerance)

Quality Assurance Team for Radiation Oncology

Reference Air Kerma Rate

Risicolnventarisatie en Analyse Straling (Risk Inventory and Analysis for Radiation ) 
http://www.doi.org/10.25030/ncs-030 This NCS report has been downloaded on 26 Apr 2023

RO

RT

RTT

SPCR

SPS

TLD

TPS

TRAK

US

\section{Radiation Oncologist}

Radiation Therapy

Radiation Therapy Technologist

Source Position Check Ruler or Source Step Viewer

Source Position Simulator

Thermo Luminescent Dosimeter

Treatment Planning System

Total Reference Air Kerma

UltraSound 


\section{Contents}

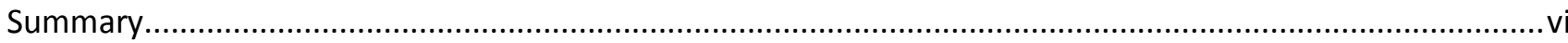

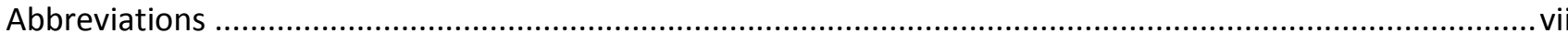

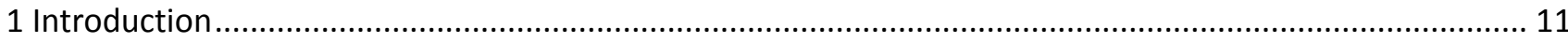

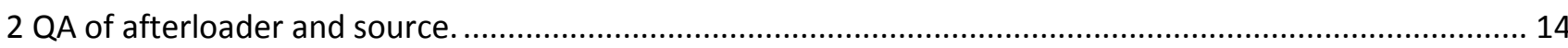

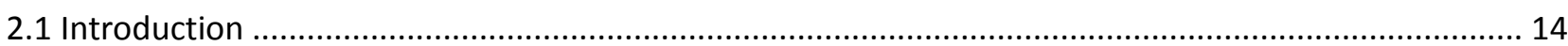

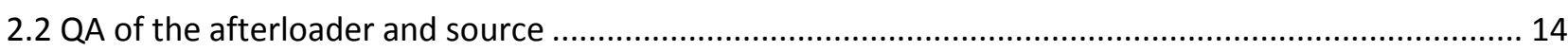

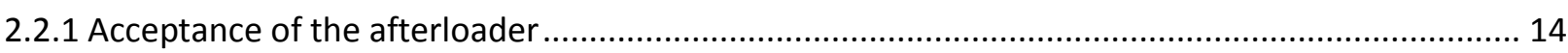

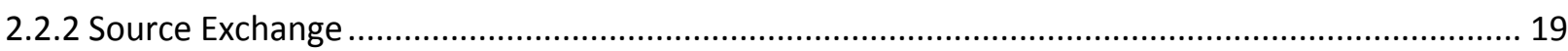

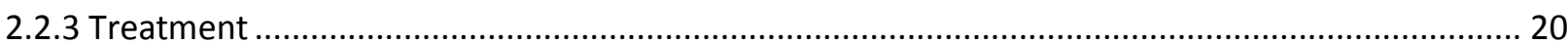

2.3 Determination of the Reference Air Kerma Rate of Ir-192 sources..............................................21

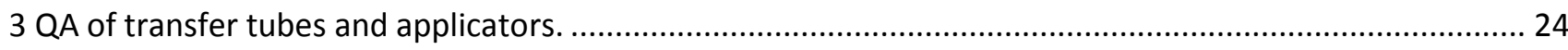

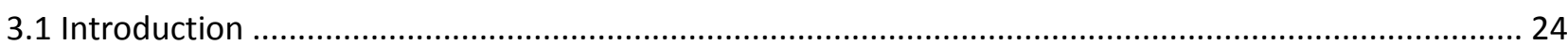

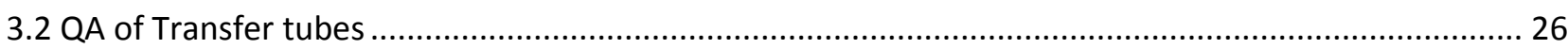

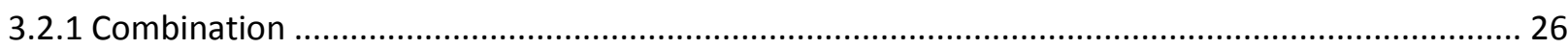

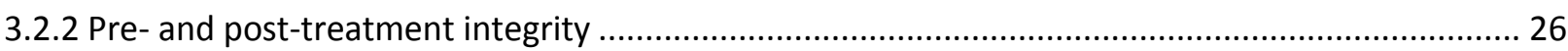

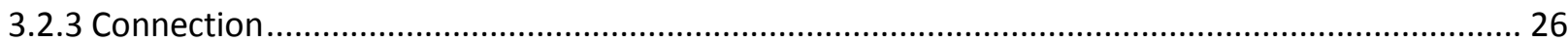

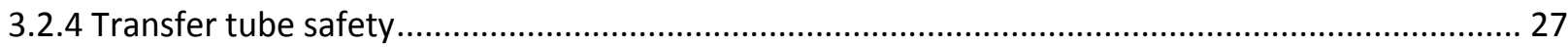

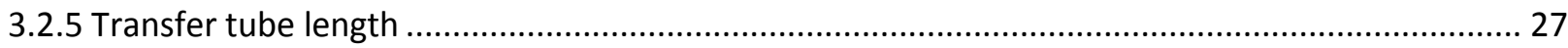

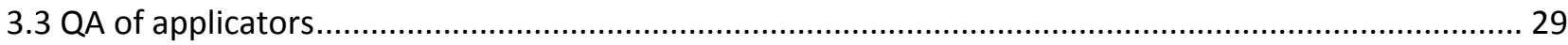

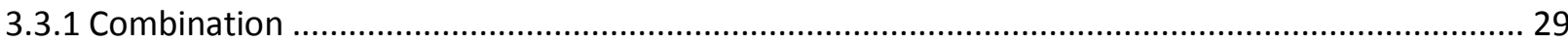

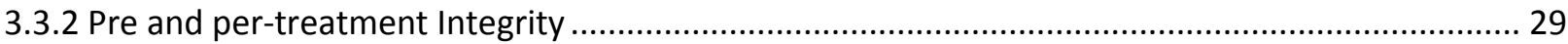

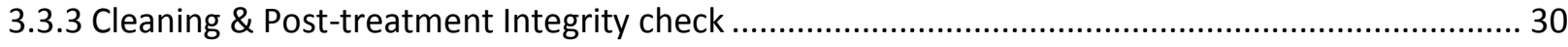

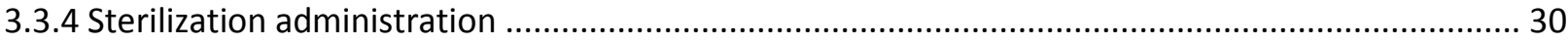

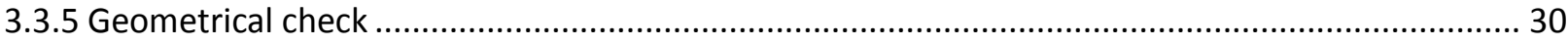

3.3.6 Additional checks/notes for specific types of applicator:.............................................. 34

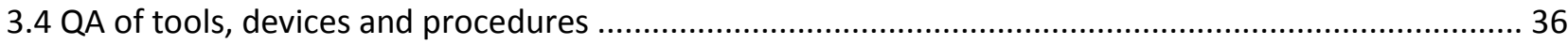

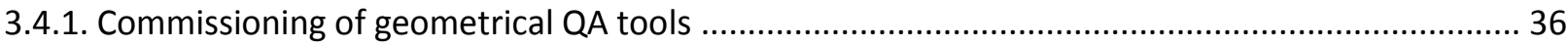

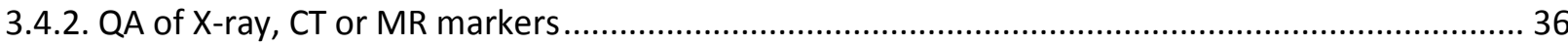

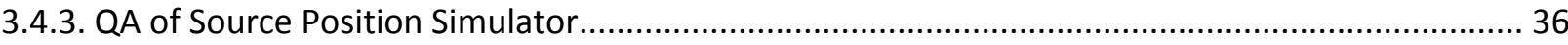

3.4.4. QA of Source Position Check Ruler ............................................................................. 37

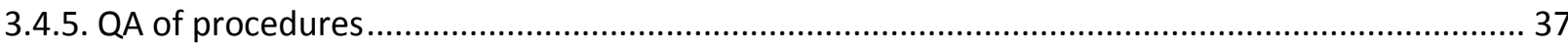

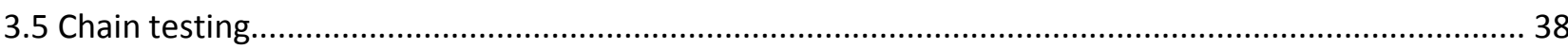

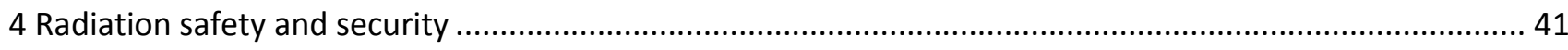

4.1 Radiation safety aspects related to Ir-192 sources............................................................ 41 
4.1.1 Radiation risk analysis for working with Ir-192 sources 41

4.1.2. Leakage radiation of the afterloader 43

4.1.3. The emergency procedure 44

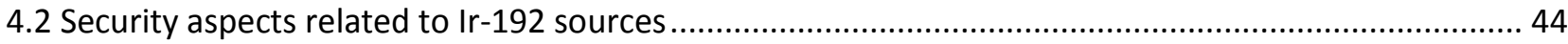

4.3 Administrative requirements for high-activity sources. ....................................................... 45

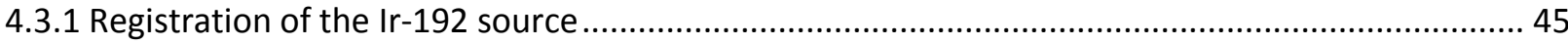

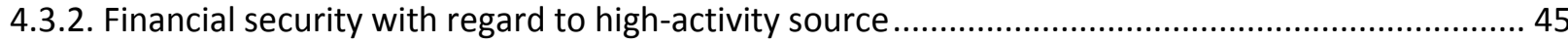

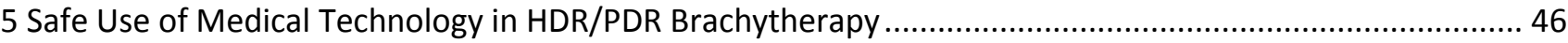

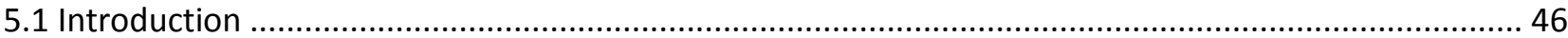

5.2 Responsibilities regarding safe use of medical technology ...................................................... 47

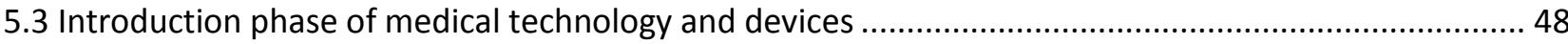

5.4 Clinical use phase: medical devices use and maintenance .................................................... 49

5.5 Decommissioning phase of medical devices............................................................................ 50

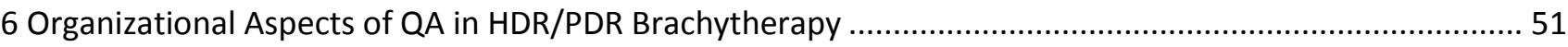

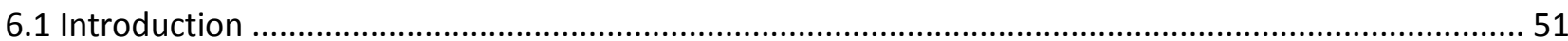

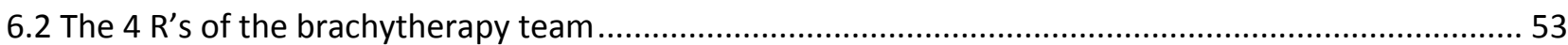

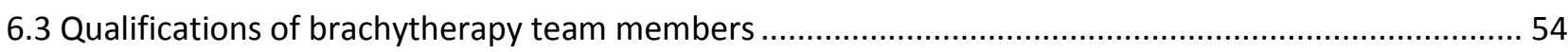

6.3.1 Specific responsibilities of the team members.............................................................. 54

6.3.2 Specific responsibilities for PDR brachytherapy ............................................................ 56

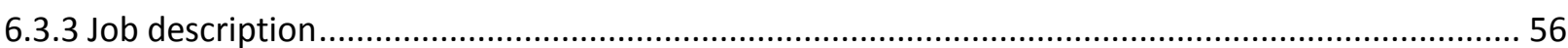

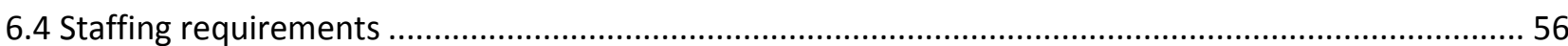

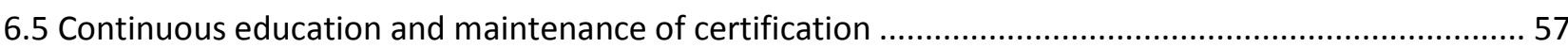

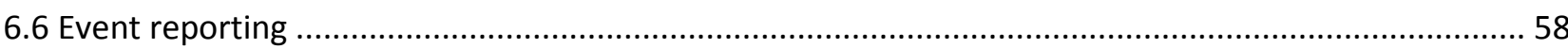

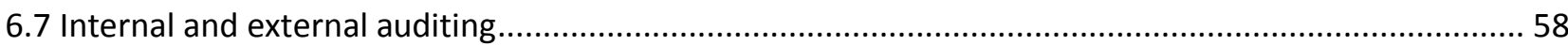

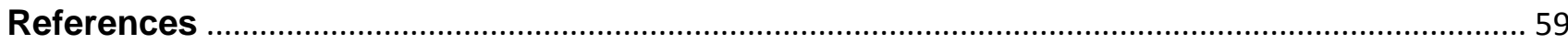

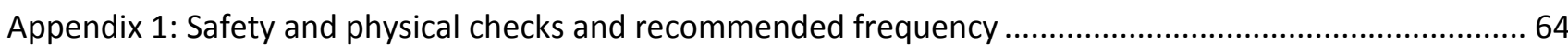

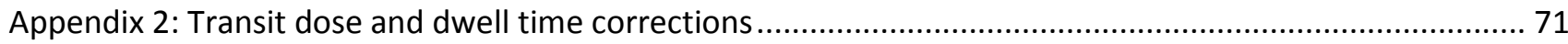

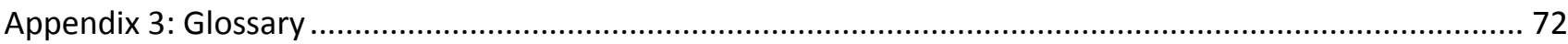




\section{Introduction}

The Netherlands Commission on Radiation Dosimetry (NCS) has published three reports on brachytherapy with Ir-192. In NCS Report 4 [1] recommendations for the specification of the source strength and the dosimetry of low dose rate (LDR) sources were given. NCS Report 7 [2] dealt with the calibration of Ir-192 high dose rate (HDR) sources. NCS Report 13 [3] gave general recommendations on the quality control (QC) of brachytherapy equipment.

Several reports have been published on safety, risk analysis and quality assurance (QA) in radiotherapy in international literature: "Safety is no accident" [4] in the United States, "Towards safer radiotherapy" in the UK [5], "Radiotherapy Risk Profile" by the World Health Organisation [6] and the report of the AAPM Task Group 100 on risk analysis methods [7]. These reports shed a light on general ways to improve the safety in radiotherapy. Other reports give more practical solutions on Quality Assurance in Brachytherapy [8-11].

The purpose of the current report is to update and partially replace the three previous reports of the NCS, resulting in a code of practice for Quality Assurance for Ir-192 Brachytherapy. Iridium wires, Cesium sources as well as beta sources for coronary brachytherapy are not used in the Netherlands and Belgium anymore so these are not discussed in this report. Other types of brachytherapy, such as lodine-implants, are dealt with in other NCS reports [12,13]. Dose calculations, a major topic in all three previous NCS-reports, are not dealt with in this report. Dose calculations according to TG43 are the international standard in brachytherapy. Abundant literature on this topic is available [14-16].

The scope of the report has evolved from source strength determination to quality assurance of the brachytherapy delivery process. This report defines this delivery process as follows (relevant chapters are indicated between brackets):

A control computer (Ch2) sends a calibrated source (Ch2) from an afterloader (Ch2) through a transfer tube (Ch3) to an intended location in an applicator (Ch2, Ch3) for an intended period of time (Ch2), in a controlled manner (Ch5, Ch6) and safe for patient and employees (Ch4, Ch5).

Imaging of the patient, delineation of target volumes and organs at risk and treatment planning are outside the scope of this report. However, only if the quality of all individual steps in preparation and delivery and the whole process chain is assured, the quality of the brachytherapy treatment can be assured.

Chapter 2 describes the QA steps to be taken on the afterloader and source during acceptance, during source exchange and before every fraction, while chapter 3 describes the QA for the transfer tubes and 
applicators. These two chapters describe the practical QA steps which have to be performed. However, while this could lead to a technically adequate treatment, modern healthcare asks for "safe equipment in the hands of a trained user in a safe environment", as stated by the Dutch "Convenant voor Veilige Toepassing van Medische Technologie" $[17,18]$. Chapter 4 describes the radiation safety and security aspects concerned with the use of high-activity (sealed) sources. Chapter 5 describes specific steps which have to be taken to comply with the advice given in the Convenant and chapter 6 elaborates on organisational aspects with regard to brachytherapy treatments with $\mathrm{Ir}-192$.

\section{Terminology in this report}

The following levels of recommendation are used throughout the report:

"must" means there is a legal obligation in Dutch and/or Belgian law;

"should" means a strong recommendation. Not abiding to this recommendation needs written argumentation and a proper alternative method to cope with the issue at hand;

"recommend" or "advise" means a mere suggestion. This recommendation can be disregarded at own will, although the subcommittee did recommend it for a reason.

Stating that a check should be done 'according to local protocol' means that there should be a clear, written protocol on how to check that particular item, including tolerance/action level and the person responsible. Since local workflow highly influences the most practical approach, the subcommittee chooses not to prescribe how to perform checks in detail. Recommended checks and frequencies depend on the equipment used, the intensity and degree of usage and local procedure.

The recommendations in this report aim for optimization of the treatment procedure by optimizing QA procedures. However, the reader should be aware that safety recommendations as described elsewhere, for instance by manufacturers, still have to be considered. In general, recommendations should not be followed blindly, but always with careful and thorough thinking.

The recommendations in this report are characterized by a minimum test frequency and an action level. An increase in test frequency or decrease in action level is required when the stability of a system is suspicious, or when a specific patient treatment method demands an exceptional accuracy. The recommended test frequencies in this report concern the regular quality control tests. Test methods and results of these tests should be documented.

Whenever in this report 4 Months $(4 M)$ is used as a recommended minimum test frequency, this is often based on the source exchange period as quality control on remote HDR and PDR afterloading equipment is often combined with a source exchange. 
An action level is defined in such a way that whenever an action level is reached, it is essential that appropriate actions are taken. The radiation equipment should not be used whenever the action level has been exceeded. On few occasions, it might be justified to use the radiation equipment clinically, even if an action level has been exceeded. Such a decision can only be taken after careful consideration by the responsible Medical Physics Expert, in consultation with the clinicians and the RTT's and should be documented in all cases.

Terminology as used by the subcommittee has been given in appendix 3 Glossary. 


\section{$2 \mathrm{QA}$ of afterloader and source}

\subsection{Introduction}

Performing good quality assurance (QA) of treatment units for brachytherapy with Ir-192 comprises quality assurance of all the steps in the life cycle of both the afterloader and the source. Some of the steps in this life cycle are, however, not under control of the user in the hospital, because they are performed by the manufacturer of the afterloader or the vendor of the source. The responsible Medical Physics Expert should nevertheless be aware of these checks. Among these steps are tensile strength of the cable, quality of the weld of the cable and source calibration ${ }^{1}$. On the source certificate some of these QA steps are briefly described.

The responsible Medical Physics Expert should also possess sufficient knowledge of the physics behind brachytherapy and radioactive sources [19].

\subsection{QA of the afterloader and source}

Quality assurance of the afterloader and source can be divided in three parts:

1) during acceptance of the afterloader system, several checks have to be performed: on the accuracy of dwell-position and dwell-time measurement; on the communication between the various devices; on safety provisions of the afterloader and in the treatment room and control room. These checks should be repeated regularly, at least when hardware or software of the afterloader has been changed.

2) during source exchange, checks have to be performed on the integrity and activity of the source. It is recommended to repeat some of the checks performed during acceptance of afterloader and treatment room with every source exchange.

3) before every treatment, checks have to be performed to decrease the chance of malfunctioning of the apparatus during the treatment.

Appendix 1 gives a list of all the recommended checks with suggested testing method, tolerances and frequencies.

It is important to document who performed which checks when and the results obtained.

\subsubsection{Acceptance of the afterloader}

Goal of acceptance is to establish that the equipment complies with the specifications given by the vendor as well as with the requirements specified by the user and authorities. Two critical parameters to be tested

\footnotetext{
${ }^{1}$ A member of the subcommittee visited Mallinckrodt Petten, manufacturer of cables and sources for Elekta, Varian and Eckert and Ziegler. The manufacturing process is highly protocolized. QA awareness of the organization is high.
} 
during acceptance of an afterloader are the accuracy of the dwell-positions and the accuracy of the dwelltimes. Other issues during acceptance of the afterloader are data transfer and safety.

\section{Accuracy of dwell-positions}

To assess the accuracy of the dwell-positions, first align the source at the reference position in the designated channel according to the alignment-procedure supplied by the manufacturer. The dwell-position can either be assessed visually (with a digital camera and a Source Position Check Ruler (SPCR) ) or radiologically (with film or other devices).

When the source is aligned to the reference position check that the source returns to this same position after returning to the safe several times, to estimate the reproducibility of the source position. Reproducibility of the position between repeated transfers should be better than $0.5 \mathrm{~mm}$. The accuracy of the dwell-position should be assessed for the reference position as well as several other positions. These positions should be selected based on the positions during clinical treatments. In a straight catheter with straight transfer tube the positional accuracy should be within $\pm 1 \mathrm{~mm}$ at all dwell-positions. It should be considered to assess the dwell-position accuracy also for other channels than the one designated by the manufacturer with the same transfer tubes.

AAPM report 59 [11] states that in most clinical applications a positional accuracy of $\pm 2 \mathrm{~mm}$ is reasonable. It should be noted that when using bended catheters and applicators, two effects can occur that lead to decreased position accuracy: 'curving' and 'snaking'. In literature, these terms are often used interchangeably. Note that these effects can occur simultaneously.

Curving (figure 2.1) is the effect that, due to the curvature of the bended transfer tube or applicator, the actual source-path will deviate from the centre line [20].

In order to estimate the magnitude of the path deviation effect in the transfer tube it is advised to assess the source position with various bends in the transfer tube. To decrease the effects of positional inaccuracy it is advised to use the transfer tubes during treatment as straight as possible.

In certain applicators path deviations up to $6 \mathrm{~mm}$ have been found $[20,21]$. Because the path deviation effect is dependent on the type of applicator used, it has to be determined for each type of applicator. See also chapter 3. In the experience of the authors the curving effect in applicators is more pronounced than in transfer tubes. When reproducible, large deviations can be taken into account during the treatment planning process by introducing in the Treatment Planning System (TPS) the real source trajectory instead of the nominal trajectory. 


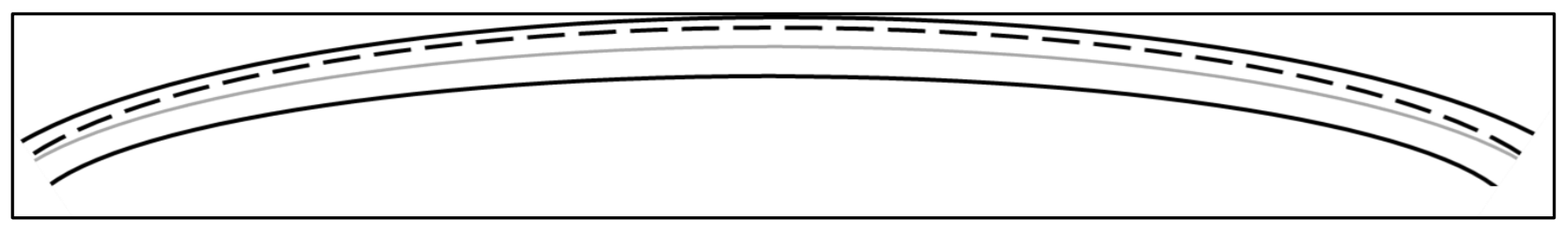

Figure 2.1: Path deviation in transfer tube: the actual source path (- - - ) deviates from the ideal source path (-).

Snaking (figure 2.2) is the effect that the source cable is curled inside the catheter when the source collides with an obstruction or the end of an applicator. There are no data in literature available on the magnitude of the snaking effect.

The detection of obstructions by the afterloader can be hampered by the snaking effect (see chapter 3 ). This is particularly important for dwell positions at the end of the source channel, where errors greater than $5 \mathrm{~mm}$ can remain undetected. Users should check what the detection threshold (or sensitivity) of their system is. Vendors should disclose this detection threshold in the operating manual of the afterloader.

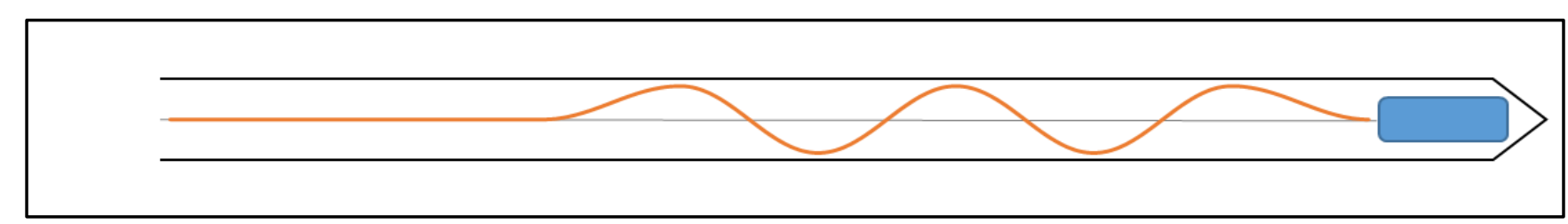

Figure 2.2: Snaking effect in applicator: the source-cable is curled; indication of obstructed applicator is inaccurate.

\section{Accuracy of dwell-times.}

The inaccuracy on the administered dwell time shall not exceed $1 \%$ or $100 \mathrm{~ms}$ whichever is greater, according to IEC 60601-2-17 [22]. This should be assessed for both small ( $\leq 1 \mathrm{~s})$, and large ( $>60 \mathrm{~s}$ ) dwell-times. To assess the accuracy of dwell-times it is important to take into account that most manufacturers include the transit time in the dwell time of the next dwell position, except for the first dwell-position $[23,24]$. Note that the clinical effects of the transit dose can be substantial (see appendix 2) [25].

Dwell-time accuracy can either be evaluated with a high speed video camera with at least 25 frames per second or by repeated measurements with an ionisation-chamber and electrometer.

Procedure with the ionisation-chamber and electrometer: first determine $I_{\text {steady }}$ by measuring for $60 \mathrm{~s}$ while the source is at the measurement-position continuously. Then collect charge on the electrometer while sending the source from the container to the measurement position for various times ranging from $0.1 \mathrm{~s}$ to $60.0 \mathrm{~s}$. Source-out time is defined on the afterloader. From this series of measurements, calculate the slope and offset. The offset is the charge collected during the transport time between the safe and the measurement position; the slope should be equal to $I_{\text {steady }}$ within $0.5 \%$ (figure 2.3 ) 


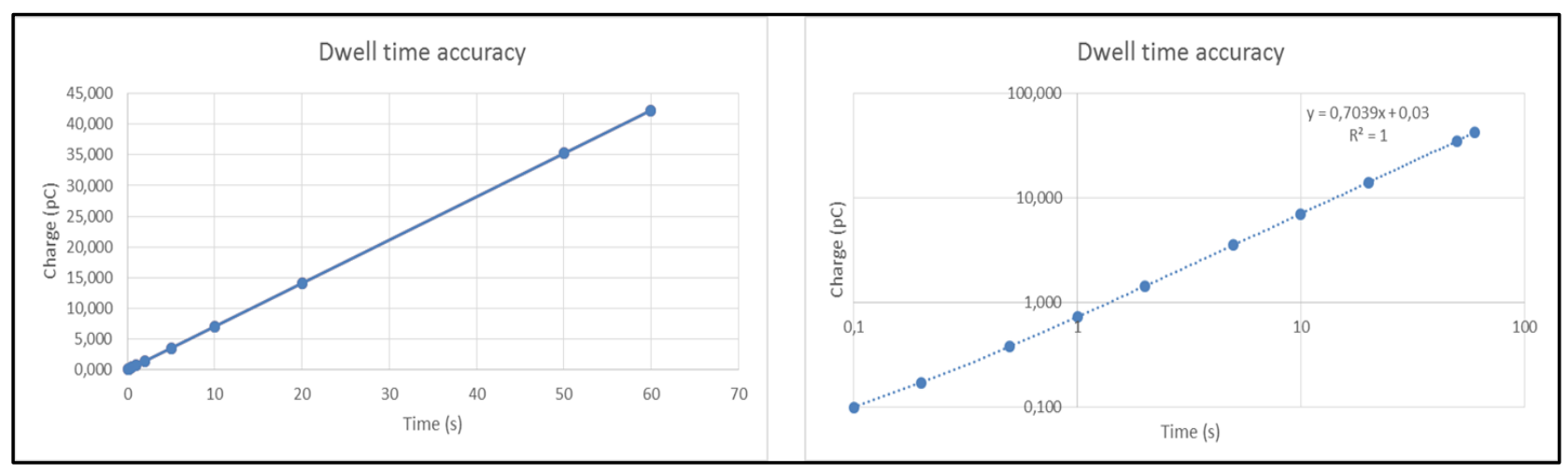

Figure 2.3: Assessment of dwell time accuracy.

\section{Communication between the TPS, the Control Computer and the afterloader}

This can be checked by sending and irradiating a complicated plan.

Check that data are correctly transferred from the TPS to the Control Computer. These data consist at least of the Total Reference Air Kerma (TRAK), the number of catheters involved and their corresponding channels, step-size, dwell-times and dwell-positions. Check that changes in Air Kerma Rate (AKR) between TPS and afterloader are accounted for accurately. Not only check that the correct values are transferred, but also that deliberately induced errors are handled correctly. Also check that useful and accurate error-messages are given.

Check that data are correctly transferred from the Control Computer to the afterloader. These data consist at least of the number of catheters involved and their corresponding channels, step-size, dwell-times and dwellpositions. Not only check that the correct values are transferred, but also that useful and accurate errormessages are given.

Tests on the safety provisions on the afterloader and in the treatment room and control room.

To ensure (radiation) safety to patients and personnel working with the afterloader, several provisions have to be taken in and around the treatment room. These provisions consist of emergency buttons both inside and outside the treatment room; door interlocks; an afterloader-generated signal indicating that the source is out of the safe; an independent external radiation monitor in the treatment room connected to a visual and audible indicator for "source is out of safe".

The afterloader itself has safety provisions that have to be tested as well: backup battery in case of power loss; check for missing or obstructed applicator. During acceptance, electrical safety and equipotential have to be tested. Even before acceptance, specifications of the manufacturer concerning e.g. floor covering and local demands concerning hygiene have to be taken into consideration. 


\section{Warning lights}

Radiation in the treatment room is indicated by warning lights. At least a warning light connected to the independent radiation monitor must be present. Additional warning lights connected to the afterloader or control computer may exist. Check that warning lights are on/off when radiation from the source is present/absent.

\section{Room monitor}

Radiation in the treatment room is monitored by a radiation monitor. Check that radiation levels are correctly monitored when radiation is present/absent.

\section{Patient Camera and Microphone}

A patient in the treatment room should be monitored during treatment with a patient camera and microphone. Check the proper functioning of both camera and microphone.

\section{Interrupt button}

Pressing the interrupt button brings the source back into the safe. Check the proper functioning by pressing the interrupt button. Check that treatment continues properly after releasing the interrupt.

\section{Door interlock}

Opening the door during treatment brings the source back into the safe. Check the proper functioning by opening the door. Check that treatment can be continued properly after closing the door.

\section{Emergency stop}

Pressing an emergency stop brings the source back into the safe. Check the proper functioning by pressing and releasing the various stops. Check that treatment can be continued properly after releasing the emergency button. Check that a proper report of the partly delivered treatment is generated.

\section{Power loss}

When power gets lost, the treatment is interrupted immediately. A backup battery provides the power to bring the source back into the safe. Check the proper functioning by simulating a power loss. Check that treatment can be continued properly after the power has been restored. 


\section{Leakage radiation of afterloader}

The amount of radiation leakage of the afterloader must comply with national and international regulations. Often, maximum radiation levels are set at $10 \mathrm{~cm}$ from the surface of the afterloader.

Some of the tests performed at acceptance of the afterloader should be repeated regularly. Appendix 1 describes the suggested minimum frequency for these tests. For practical reasons the local protocol could prescribe these test to be performed at each source exchange.

\subsubsection{Source Exchange}

The manufacturer of the afterloader supplies a method to safely unload and load the source in the afterloader. Preferably, this is performed by a service engineer of the afterloader vendor. For security reasons the hospital should devise a policy whether the source-exchange may be performed by this service-engineer of the vendor with or without a hospital-employee present at all times. Alternatively, an engineer of the hospital could be trained to perform source exchanges.

Some manufacturers of afterloaders demand those who perform source-exchanges to be formally trained and provide certificates to trained personnel. When this is the case, the responsible Medical Physics Expert has to assure that all personnel performing the source exchange are adequately trained and have this certificate required by the manufacturer.

The license of the institute requires all personnel performing source exchanges to have the appropriate level of radiation safety training as well. The responsible Medical Physics Expert has to check that all personnel performing the source exchange have the mandatory certification. This is also necessary for personnel of the afterloader manufacturer performing the source exchange [18].

During source exchange the service-engineer performs a number of checks required by the manufacturer of the source and/or the manufacturer of the afterloader. The responsible Medical Physics Expert should be familiar with the checks and determine whether additional tests have to be performed by an employee of the hospital. When the checks performed by the manufacturers service-engineer are part of the quality assurance chain of the hospital, it has to be assured that the service report has been received before releasing the source for clinical use. 


\section{Contamination test}

Source, source cable, dummy and dummy cable should be free of radiation contamination. Maximum dose levels are set in national regulations as well as the CE-mark of the afterloader. This test is performed during source-exchange on both the old and new source with a wipe test.

\section{RAKR measurement}

Each new source comes with a certificate from the manufacturer specifying the Reference Air Kerma Rate (RAKR). This certificate should describe the procedure followed during RAKR determination, as well as an estimate of the accuracy of the determined RAKR. After the source exchange, and before clinical use, the source strength must be measured according to the local protocol and compared against the value stated on the source certificate. When the measured value deviates more than $5 \%$ from the specified value on the certificate, the source must not be taken into clinical use and the source manufacturer should be contacted. Discrepancies larger than 3\% should be investigated [26]. Based on local experience, the local protocol can foresee additional more stringent action levels. When traceability is guaranteed for both the value on the certificate as the measured value, either the measured value or the value on the certificate can be used as reference for treatments. With regard to the RAKR-values it is important to realize that both values are measured with a traceable calibrated instrument, but that the methods and procedures of these measurements are not accredited, neither for the manufacturer nor for the local protocol. The local protocol should prescribe which value should be used and entered in the control computer.

A qualified person must check the RAKR value entered in the control computer, according to local protocol. The source should not be clinically used before this check has been performed. Name, date and time of the person performing this check should be documented. Depending on the local situation the RAKR should also be entered in the TPS.

Registration of the RAKR data is recommended for trend-analysis to detect drift in the measurement chain as well as changes at the manufacturer.

Local protocol should prescribe how changes due to daylight saving time should be accounted for. It is advisable to use the same time throughout the year [26].

In chapter 4 an overview is given of the administrative issues related to the source exchange that have to be applied in Belgium and the Netherlands.

\subsubsection{Treatment}

On each day a treatment is performed, a quick check of the whole treatment chain is recommended. In this check data transport, source decay, source position accuracy, functioning of door-interlock, warning-light and audio-visual communication systems and functioning of a dedicated imaging system can be checked within 
several minutes. For example, a plan with one treatment position in one catheter could be sent from the planning system to the treatment console and then irradiated on the SPCR, thus checking several of the aforementioned parameters. Next to this performance check of the treatment equipment a check of the availability of the emergency kit, emergency procedures and survey meter could be performed.

\subsection{Determination of the Reference Air Kerma Rate of Ir-192 sources}

ICRU reports 38 [27] and 58 [28] recommend the dosimetric specification of gamma sources in terms of Reference Air Kerma Rate, which can be determined by several methods: with an 'in-air' measurement technique, with a dedicated solid phantom or with a well-type ionisation chamber. Specification 'in-water' has the advantage of specification to the desired medium, but the method is currently not widespread. The in-air measurement has the advantage that it determines the source strength in the desired quantity: Air Kerma Rate at a certain distance, from which the Reference Air Kerma Rate is calculated. The downside of this method is that the typical signals are very low and that the resulting uncertainty of the Reference Air Kerma Rate is high. Other disadvantages are the uncertainties in the distance between the catheters , uncertainties in the position of the source inside the catheters and uncertainties in the determination of the various correction factors that have to be applied [8].

An advantage of the solid phantom method is the accurate positioning which results in a more precise measurement. A scatter free environment is not needed for the measurement. However several correction factors, specific for the phantom and measurement setup, have to be used. The uncertainty in the determination of these correction factors can be substantial.

In this report, the well type chamber technique is the recommended method for source calibration because it is an accurate, fast, reproducible and easy method. Well chambers are worldwide commercially available and nowadays calibration for well type chambers for Ir-192 at primary standard laboratories are widely accessible. It is recommended to calibrate the chamber every two years. Disadvantage of the well type chamber technique is the necessity of a low scatter environment and the long time it takes for a well chamber to reach temperature stability. IPEM published a useful Code of Practice on the determination of the Reference Air Kerma Rate with a well-type chamber [26]. The Reference Air Kerma Rate $\dot{K}_{\text {ref, }}$ with unit $\mathrm{mGy} / \mathrm{h}$ is defined as the Air Kerma Rate at a distance of $1 \mathrm{~m}$ in air, corrected for air attenuation and scattering.

$$
\dot{K}_{\text {ref }}=I_{\text {raw }} \cdot f_{\text {elec }} \cdot k_{\text {ion }} \cdot k_{\text {sg }} \cdot k_{\text {dec }} \cdot k_{p, T} \cdot N_{R A K R}
$$


where:

$\mathrm{I}_{\text {raw }}=$ electrometer current reading in $\mathrm{nA}$;

$\mathrm{f}_{\text {elec }}=$ electrometer correction factor to convert the current readings to the actual current;

$\mathrm{k}_{\text {ion }}=$ ion-recombination correction factor determined with the hospital source at the time of the measurement

$\mathrm{k}_{\mathrm{sg}}=$ the source geometry factor corrects for change of the well chamber response because of differences in source type between the calibration source and the hospital source.

$\mathrm{k}_{\mathrm{dec}}=$ decay correction factor to correct the measured Reference Air Kerma Rate to a reference time

$\mathrm{k}_{p, T}=$ correction factor for temperature $\mathrm{T}$ and pressure $\mathrm{p} ;$

$\mathrm{k}_{p, T}=\frac{(\mathrm{T}+273.15)}{\left(\mathrm{T}_{0}+273.15\right)} * \frac{p_{0}}{\mathrm{p}}$

$\mathrm{N}_{\text {RAKR }}=$ Reference Air Kerma Rate calibration factor $\left(m G y \cdot m^{2} \cdot h^{-1} \cdot n A^{-1}\right.$ ) (at $\mathrm{p}_{0}$ and $\mathrm{T}_{0}$ ), from the standard laboratory

Attention has to be paid to the following:

- A dedicated insert should be used during measurement as well as during calibration of the well-chamber, to ascertain a reproducible source position;

- Measurements have to be performed in a low scatter environment: the chamber has to be positioned at least $1 \mathrm{~m}$ from wall or floor;

- The measurements have to be taken with the source at a fixed position, preferentially at the position of maximum response. When measuring in a fixed position, correction for source transit is not necessary;

- $\quad$ Calibration values as given by the standard laboratory depend on the source strength. For high Air Kerma Rates recombination corrections should be considered, depending on the calibration procedure in the standard laboratory. The Dutch Metrology laboratory, VSL, uses a source which has decayed to a strength at which the level of recombination is too low $(<0.1 \%)$ to be measured accurately. Therefore it is neglected and an additional uncertainty is added to the total uncertainty. For clinical application the user should evaluate whether the recombination of the chamber for the maximum source strength is of a clinically relevant level.

- $\quad$ The calibration value as given by the standard laboratory is only valid for a specific source type. The differences between various source types can be significant and can be corrected for if a source geometry factor, $\mathrm{k}_{\mathrm{sg}}$, for the source types in question and well chamber is available.

- The interior parts of the well chamber should be allowed to stabilize to the temperature of the room where the measurement is performed. Especially if considerable differences exist in 
temperature between the storage room of the chamber and the room where the measurement is performed, it can take several hours to reach this temperature.

- Drift in chamber response over a prolonged time has been reported [8]. To assess this drift the response of the chamber to a calibration source can be used. This drift can also be assessed by performing an exit measurement before unloading the old source. When a deviation larger than $0.5 \%$ between expected and measured value is found the measurement chain should be checked. - $\quad$ By performing measurements of ionisation currents instead of measurements of accumulated charge or by using the internal timer of the electrometer, inaccuracies caused by source transit can be overcome. 


\section{QA of transfer tubes and applicators}

\subsection{Introduction}

\section{Commissioning}

After receiving new applicators or transfer tubes/source guide tubes ${ }^{2}$, proper commissioning is mandatory. CE certification should be verified, and the manual consulted. See chapter 5 for the Dutch Convenant on such issues. Next, make sure that the QA tools are functioning properly (section 3.4). Subsequently perform appropriate geometrical checks (section 3.2 and/or 3.3). Finally, a chain test for the combination of afterloader, transfer tube and applicator, including geometrical applicator parameters in the TPS, is strongly recommended for the combinations that may be used clinically (section 3.5). This chain test focuses on the geometrical aspects, although the dosimetric aspects should be considered as well. For single-use devices (such as e.g. needles) it is recommended to regularly check one randomly chosen device per batch.

\section{Regular use}

Over time, both applicators and transfer tubes may show (signs of) mechanical wear and tear due to, among others, cleaning, disinfection and sterilisation, aging of the fabric, wear due to movement of the source and check cable (friction), bending of the tubes and (dis)connecting the transfer tube from the afterloader and applicator(s). Worldwide there have been several reports of damaged applicators or transfer tubes and poor connectivity between transfer tubes, afterloader and applicator. Wearing of material may theoretically compromise hygiene or radiation safety if the source cannot retract due to a faulty connection or biological contamination in either transfer tubes or applicator.

Therefore, it is highly recommended to check both applicators and transfer tubes carefully for integrity, leakage and proper connectivity before and after each use.

\section{Life expectancy}

Usually, the manufacturer provides instructions for recommended checks along with an average life expectancy of the various parts. However, it is the user's responsibility to ascertain good functioning, irrespective of the expected life span indicated by the manufacturer. Since wear and tear depend both on intensity and degree of usage as well as on cleaning, disinfection and sterilisation frequencies, these factors may be considered along with the date and time since purchase of the applicator or transfer tube. If the manufacturer expresses the life expectancy in years, rather than in number of treatments or usage, it is

\footnotetext{
${ }^{2}$ in this chapter 'transfer tubes' will be used for brevity
} 
advised to check which assumptions, for instance number of treatments per week, are made for determining this period.

\section{Dominant factors determining life expectancy}

The NCS subcommittee considers the dominant factors determining deterioration to be:

The number and type of cleaning, disinfection and sterilisation cycles

Wear and tear due to repeatedly (dis)connecting the various parts, in particular for PDR treatments during which (dis)connections are made frequently

Wear and tear due to source and check cable movement

Aging of materials (physical aging, chemical aging, thermal aging, radiation exposure)

Storage conditions

Which factor is dominant depends on local procedures and usage of the particular equipment.

\section{Recommended checks}

The recommendations in this report aim for minimization of the occurrence of obstructions or other malfunctioning during treatment execution. Safety recommendations as described elsewhere, for instance by manufacturers, also apply.

Stating that a check should be done according to 'local protocol' means that there should be a clear, written protocol on how to check that particular item, including tolerance/action level and who is responsible. Since local workflow and equipment highly influence the most practical approach, the subcommittee chooses not to prescribe how to perform checks in detail.

Recommended checks and frequencies depend on the equipment used, the intensity and degree of usage and local procedure.

The manufacturer's manual can be a starting point to set up local procedures, however, well-substantiated deviations can be considered. The responsibility of having a clear and comprehensive protocol lies with the Medical Physics Expert. Although tests can be performed by RTTs or medical physics engineers, in case of problems or doubt, a Medical Physics Expert responsible for brachytherapy should always be consulted.

\section{De-commissioning}

The parts that are no longer used should be marked as decommissioned, and stored separately from the parts that are in active clinical use. In addition, if parts are unacceptably damaged, this should be documented and communicated to the vendor if this is unexpected. 


\subsection{QA of transfer tubes}

The aim of QA of transfer tubes is the assessment of the correct length and integrity of the tubes.

For the transfer tubes in use it is important to guide and support them to prevent from kinking. Guide the tubes from the afterloader to the applicator as straight as possible. Consider using a special support tool in case numerous tubes are being used (e.g. prostate treatment).

To avoid bends or kinks, it is recommended to store the tubes fully elongated, either horizontally or hanging vertically, when disconnected from the afterloader.

It is recommended to have a reliable estimate on the frequency of use of each transfer tube in relation to the value used to calculate life expectancy.

\subsubsection{Combination}

Aim: use of the right combination of afterloader-transfer tube-applicator.

Background: different transfer tubes may have different lengths and connectors. It is important to use a combination of correct length and connector, to avoid damage, source obstruction and wrong dwell positions.

Test: check that the transfer tube matches the chosen applicator.

Frequency: before each treatment fraction.

Tolerance: must pass.

\subsubsection{Pre- and post-treatment integrity}

Aim: to avoid using damaged/dirty transfer tubes.

Pre-treatment test: visually inspect that all parts (tubes and connectors) are clean and intact.

Post-treatment test: visually inspect that all parts are biologically clean, and inspect for indication of weakening, bending, kinks, corrosion, discoloration or general deterioration.

On indication: cleaning manually or using ultrasound if the connector is contaminated internally. Frequency: before and after each treatment fraction.

Tolerance: must pass.

\subsubsection{Connection}

Aim: to assure proper fixation.

Background: all types of afterloaders have a mechanism to lock the transfer tube to the afterloader. This lock prevents the transfer tube to be released from the afterloader during treatment.

Test 1: check proper connection between afterloader and transfer tubes, and check that the lock only closes when all transfer tubes needed are inserted properly. 
Test 2: check connection between transfer tubes and applicator (locked connector).

Test 3: check both connections for play and strength.

Frequency: before each treatment fraction.

Tolerance: must pass.

\subsubsection{Transfer tube safety}

Aim: to ensure the functioning of Source Block or Missing Applicator.

Background: There should be no obstruction within the transfer tube, and if there is no applicator connected to the tube it should be impossible to send out the active source.

Test 1: check that, with applicator connected, there is no obstruction.

Test 2: check that, without applicator connected, a check cable or wire (e.g. old check cable) is blocked at the end of the transfer tube.

Frequency: annually or on indication.

Tolerance: must pass.

\subsubsection{Transfer tube length}

Aim: to check transfer tube length.

Background: the length of the transfer tube determines the position of the source in the patient.

Test: measure total length using tape measure (flat surface), see figure 3.1 for an example.

Or, measure the reference length by performing a source position check with the Source Position Check Ruler (SPCR), Length Gauge Tool, suitable phantoms, or autoradiography. Compare the measured total length with nominal total length and keep record of this measured length.

If transfer tubes have not been used for more than 12 months, perform visual and elaborate inspection. Frequency: annually

Tolerance: $\pm 1 \mathrm{~mm}$ 


\begin{tabular}{|c|c|c|}
\hline What & How & Frequency \\
\hline $\begin{array}{l}\text { Check that combination of } \\
\text { afterloader, transfer tube and } \\
\text { applicator is correct and has been } \\
\text { commissioned } \\
\text { (“Is this the right combination?") }\end{array}$ & Local protocol & $\begin{array}{l}\text { Before each } \\
\text { treatment fraction }\end{array}$ \\
\hline $\begin{array}{l}\text { Visual check } \\
\text { (“Are they clean and } \\
\text { undamaged?") }\end{array}$ & $\begin{array}{l}\text { Visually check transfer tube and connectors. } \\
\text { Ensure there is no visible damage, } \\
\text { contaminants nor kinks present. }\end{array}$ & $\begin{array}{l}\text { Before each } \\
\text { treatment fraction }\end{array}$ \\
\hline $\begin{array}{l}\text { Ensure correct connection } \\
\text { between transfer tube and } \\
\text { applicator and afterloader } \\
\text { ("Are they properly secured?") }\end{array}$ & Local protocol & $\begin{array}{l}\text { Before each } \\
\text { treatment fraction }\end{array}$ \\
\hline Visual inspection & $\begin{array}{l}\text { Visually inspect all items: } \\
\text {-Biological contamination, integrity, kinking. } \\
\text { If contaminated, clean tubes and connections } \\
\text { according to manufacturer's protocol. } \\
\text { If unacceptably damaged, document this } \\
\text { (photograph) and decommission transfer } \\
\text { tube. }\end{array}$ & $\begin{array}{l}\text { After each treatment } \\
\text { fraction }\end{array}$ \\
\hline Elaborate inspection & $\begin{array}{l}\text { Measure reference length with a tape } \\
\text { measure or by performing source position } \\
\text { check. } \\
\text { Inspect connectors and check that } \\
\text { obstruction or missing applicator is detected. }\end{array}$ & Annually \\
\hline
\end{tabular}




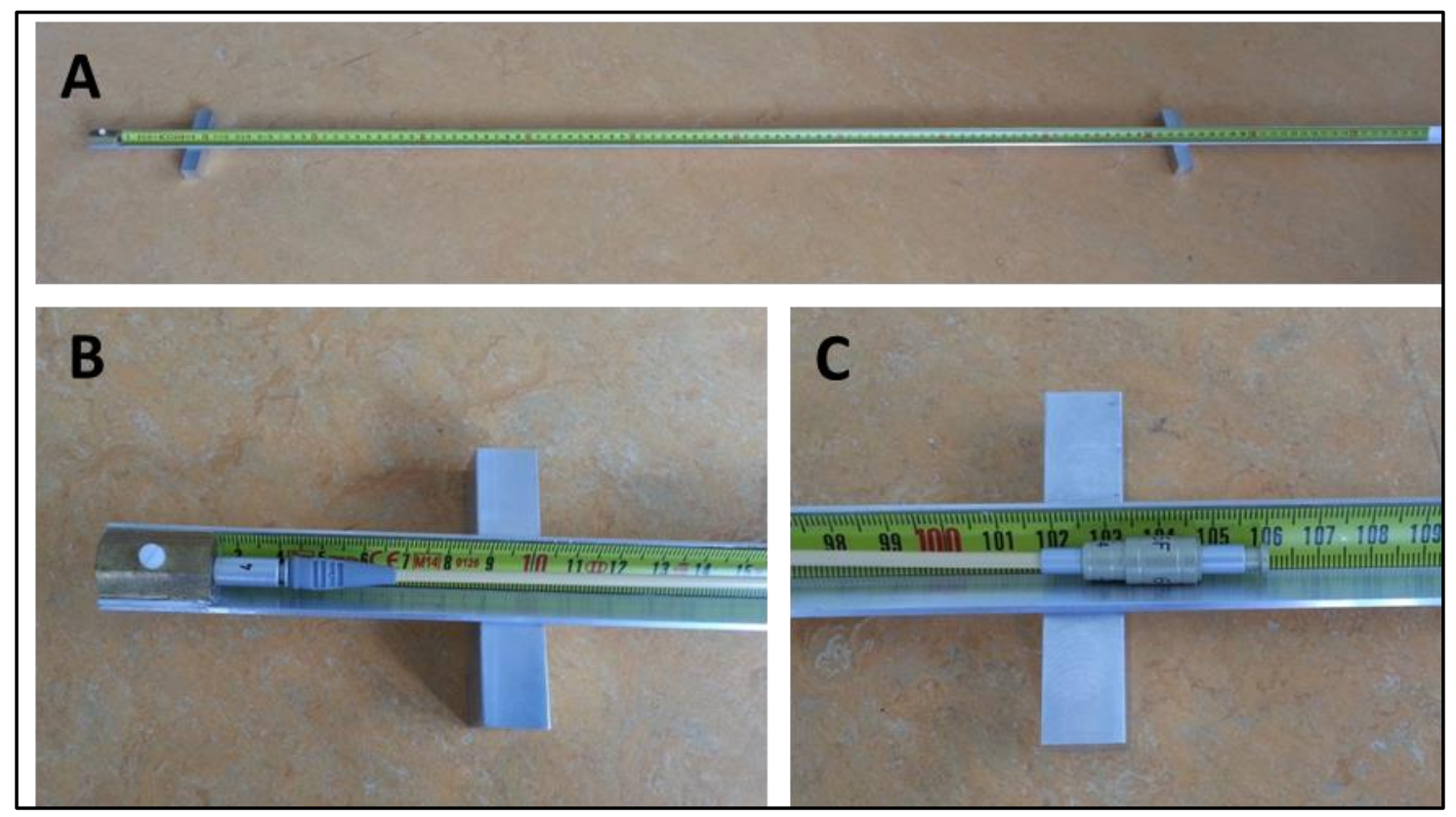

Figure 3.1: Illustration of a (home-made) measurement tool for Transfer Tubes. A: total tool, B: Mimicking connection part of the afterloader with indexer ring, C: reference length to be determined.

\subsection{QA of applicators}

In general, applicators can be divided in a few types: rigid (gynaecological) applicators with a predefined geometry, flexible or rigid needles and flexible tubes. Each type has, depending on its composition, different demands regarding QA.

First we describe the checks in general, followed by additional notes for some specific types of applicators. Instead of 'applicator', please read: 'applicator/needle/flexible tube', whichever is/are appropriate.

For commissioning of new applicators, the tests in sections 3.3.2 and 3.3.5 should be performed. In addition, the administration including cleaning and sterilization (section 3.3.4) should be set up.

\subsubsection{Combination}

Aim: use of a valid combination of afterloader-transfer tube-applicator.

Background: different applicators may require transfer tubes of specific length or connectors. It is important to use a combination of correct length, to avoid damage, source obstruction and wrong dwell positions. Test: check that the proper transfer tube is taken for the chosen applicator.

Frequency: before each treatment fraction.

Tolerance: must pass.

\subsubsection{Pre-and per-treatment integrity}

Aim: to avoid using damaged/dirty applicators. 
Background: during the sterilization process the applicator can be damaged. In addition, with certain types of applications (e.g. using sharp needles) the tubes that are already in situ may become damaged.

Test 1: visually inspect mechanical integrity and indications of damage, cracking, discoloration, or general deterioration. Especially the glued parts need attention. Note that this needs to be done under sterile conditions.

Frequency: before inserting the applicator.

Test 2: when needed, insert a guide wire through possible damaged tubes and check by wiping the extracted wire for possible contamination (e.g. blood).

Frequency: before start of treatment.

Tolerance: must pass.

\subsubsection{Cleaning \& post-treatment Integrity check}

Aim: to clean applicators and check integrity.

Background: due to the differences in compounds used, cleaning should be done according to the manuals, to avoid damage of applicators.

Test 1: clean applicator according to manufacturer's protocol.

Test 2: visually inspect mechanical integrity and for possible damage, especially of joint (glued) parts. Frequency: after removal of the applicator.

\subsubsection{Sterilization administration}

It is recommended to have a proper administration of the number of cleaning, disinfection and sterilisation cycles for each applicator.

\subsubsection{Geometrical check}

Aim: to ensure that the first (most distal) dwell position, as reconstructed with imaging (X-ray/CT/MR/US), corresponds to the true physical location of the first dwell position.

Background: depending on the reconstruction method used, the location of the reference point on Xray/CT/MR/US can be different from the first dwell position. In the TPS, the reconstructed reference point must be coupled to the first dwell position. For instance, if a thin wire up to the end of the channel is used as a CT-marker, the distance from the tip of this wire to the first dwell position must be known to determine the first dwell position in the TPS.

Whenever possible, it is advised to check source dwell positions using the radiation of the active source (autoradiography). This can be done for example by film or other high resolution detectors. 
Test: determine the first dwell position and check that it corresponds to the position at commissioning and the value in the TPS, using local protocol. Preferably, use the active source with autoradiography, but (3D) X-ray imaging in combination with the dummy source can be allowed, provided that the relation between the position of the dummy source and the active source under clinical conditions (e.g., differences in offset and cable snaking) is known. See also section 3.4.

Frequency: annually

Tolerance: $\pm 1 \mathrm{~mm}$

Figures 3.2 and 3.3 show two examples for a geometrical verification of the first dwell position and the source step size. For the use of this information in clinical practice, see for instance the paper of Hellebust et al on recommendations from the Gyn GEC-ESTRO working group of applicator reconstruction in 3D based treatment planning of cervix cancer brachytherapy. In the appendix of that paper an applicator reconstruction catalogue is given, with examples of clinical practice [29].

Note: if applicators have not been used for more than 12 months, perform additional visual inspection. Note: in principle, the geometrical check may depend on the channel used for verification. 


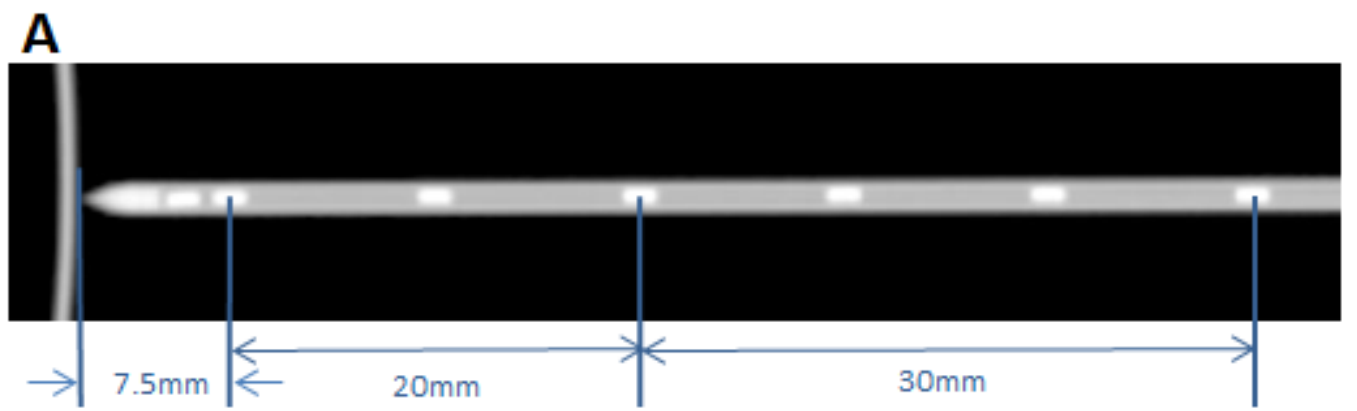

B
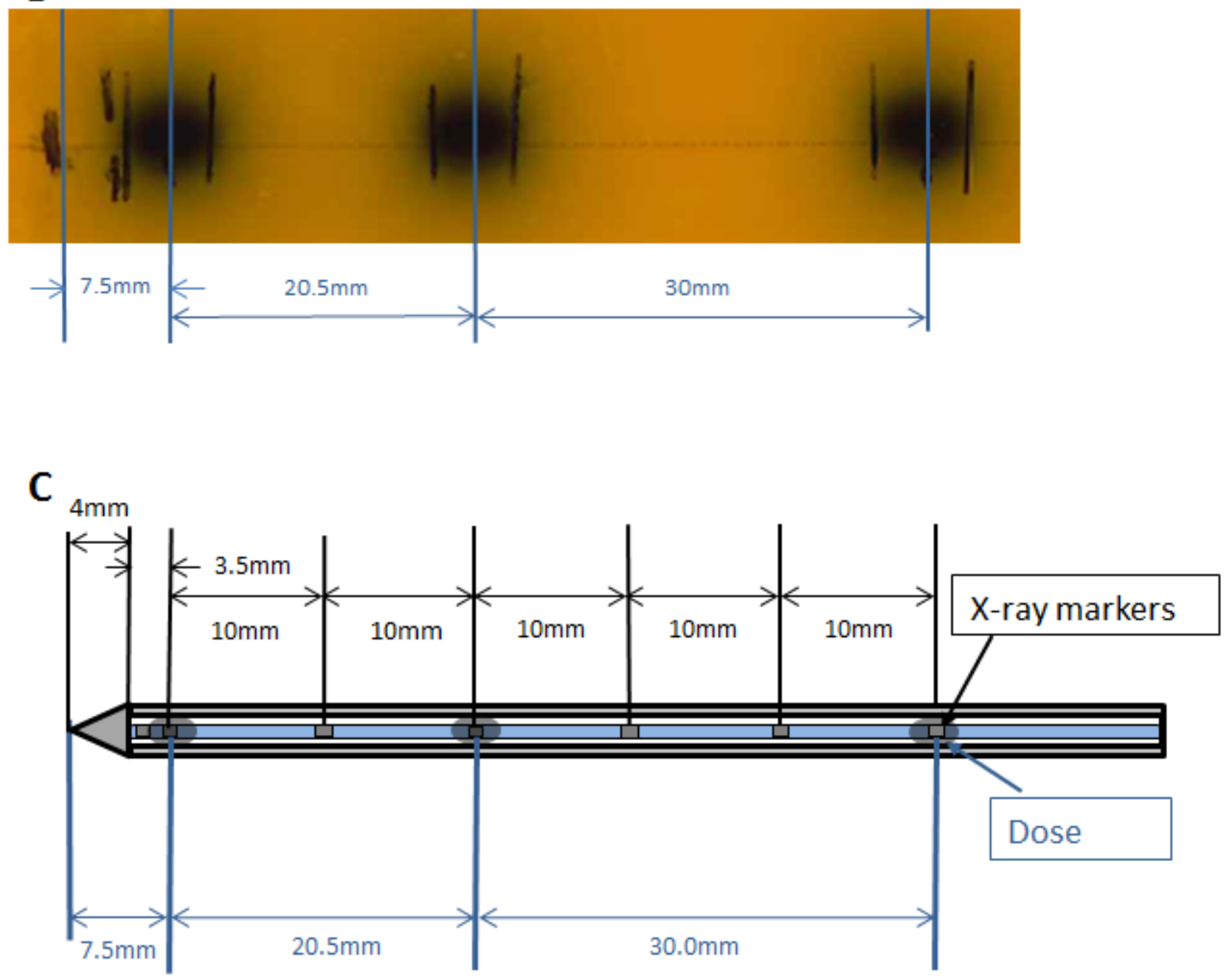

Figure 3.2: Illustration of some steps of the commissioning/QA of a Varian $250 \mathrm{~mm}$ needle used for prostate brachytherapy. The needle is fixed on EBT-film, with a lead wire perpendicular to the tip of needle.

A: An X-ray image is made, with dummy marker inside the needle, and imported in the planning system. The distances are determined with the measure function in the TPS

B: irradiated EBT film with dwell positions at 130, 128 and $125 \mathrm{~cm}$ (= first dwell position and 20 and $50 \mathrm{~mm}$ from the first dwell position), dwell time 10 seconds.

C: Schematic drawing with resulting distances from both the radiological (A) and dosimetrical (B) measurements. This is compared with the specifications of Varian and the distances used in the TPS. 
A

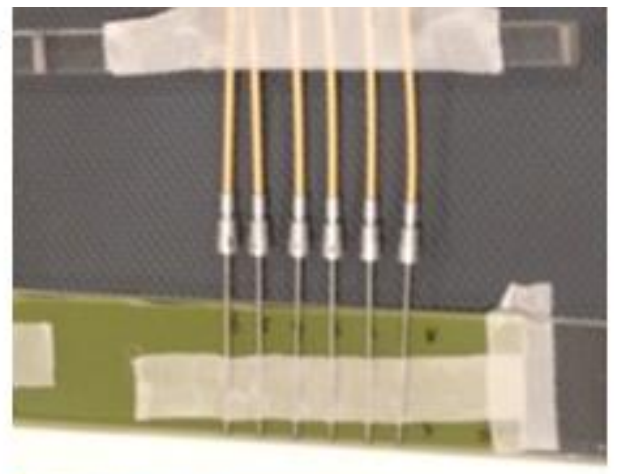

B

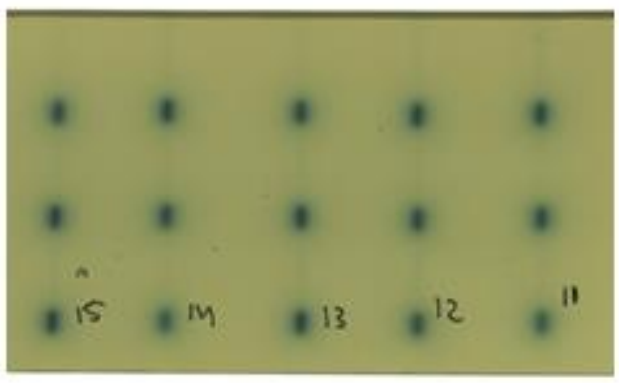

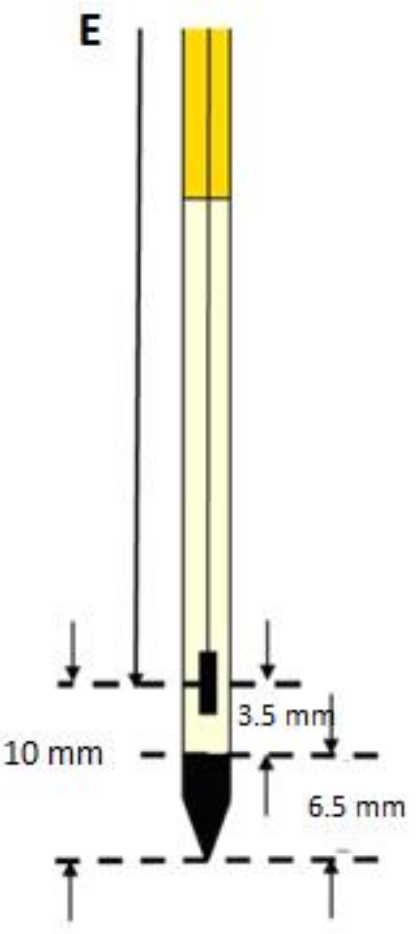
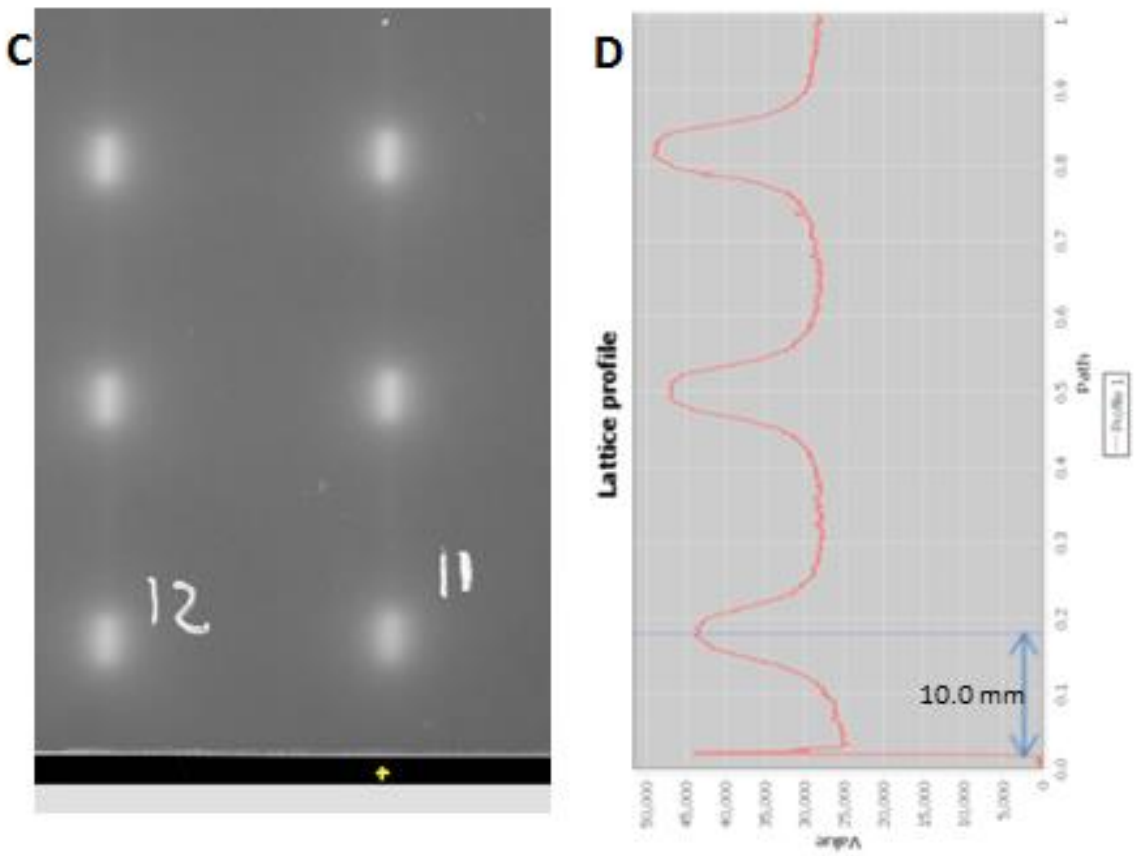

Figure 3.3: Illustration of commissioning/QA of Elekta interstitial, rigid, sharp needles.

A: Six needles, fixed on an EBT film, all fitted against the edge of a rigid structure.

B: Exposed EBT film, indicating the irradiated dwell positions (1, 5 \& 9; first dwell position and 20 and $40 \mathrm{~mm}$ from the first dwell position) (only 5 needles are depicted here)

C: EBT film imported in visualisation and measurement software to determine the relative distances (D)

D: Greyscale profile of needle 11, indicating the offset from the needle tip and relative distances of the three source positions. The edge of the film and the tip of the needles coincide (A) and are indicated by the steep rise in the profile at the bottom.

E: Schematic drawing of the different values from the Elekta manual as used in the treatment planning system. These are compared with the measured values (D). 


\subsubsection{Additional checks/notes for specific types of applicator}

Gynaecological applicators with rigid channels

Cleaning \& integrity check: some types of applicators are known to develop micro-fractures. For these applicators, it is recommended to check for hair cracks by applying slightly elevated air pressure (max. 0.2 bar), while submerging the applicator in water.

Flexible needles for interstitial/intra-cavitary use

Combination and sterilisation: in particular for flexible needles, it is recommended to check the length of a random combination of needle and transfer tube after procedures involving physical or chemical stress (e.g. sterilisation or disinfection). Changes in tube lengths due to sterilization have been reported.

\section{Flexible tubes with variable length}

For flexible tubes it is especially important to have a strict procedure for the independent determination of the first dwell position. For instance: in the case of a flexible tube of unknown length placed during operative implant, a radio-opaque wire can be used to determine the length from a reference point to the first source dwell position. Such a procedure should be tested during commissioning and always be followed clinically, in order to prevent errors due to the use of alternative reconstruction methods.

\section{Custom-designed applicators}

For custom-designed, or patient-specific applicators, without CE marking, additional procedures as demanded by the Convenant (chapter 5 ) are required. This can for instance be the case for individual moulds. Again, a strict protocol for the independent determination of the first (and other) dwell position is essential. 


\begin{tabular}{|c|c|c|}
\hline What & How & Frequency \\
\hline $\begin{array}{l}\text { Check that combination of } \\
\text { afterloader, transfer tube and } \\
\text { applicator is correct and has } \\
\text { been commissioned } \\
\text { (“Is this the right } \\
\text { combination?") }\end{array}$ & Local protocol & $\begin{array}{l}\text { Before each } \\
\text { treatment fraction }\end{array}$ \\
\hline $\begin{array}{l}\text { Visual check } \\
\text { (“Is it clean and undamaged?") }\end{array}$ & $\begin{array}{l}\text { Visually check the applicator: } \\
\text {-Mechanical integrity of joint (glued) parts, } \\
\text { colour changes, cracking. } \\
\text { Note sterility! }\end{array}$ & $\begin{array}{l}\text { Before inserting the } \\
\text { applicator }\end{array}$ \\
\hline Cleaning & $\begin{array}{l}\text { Clean applicator according to manufacturer's } \\
\text { protocol. }\end{array}$ & $\begin{array}{l}\text { After removal of the } \\
\text { applicator }\end{array}$ \\
\hline Visual inspection & $\begin{array}{l}\text { Visually inspect the applicator: } \\
\text {-Damage, mechanical integrity of joint (glued) } \\
\text { parts. If unacceptably damaged, document this } \\
\text { (photograph) and decommission applicator. }\end{array}$ & $\begin{array}{l}\text { After removal of the } \\
\text { applicator }\end{array}$ \\
\hline $\begin{array}{l}\text { Geometrical check of source } \\
\text { dwell positions }\end{array}$ & $\begin{array}{l}\text { Local protocol } \\
\text { Ensure that dwell positions correspond to values } \\
\text { at commissioning and values in TPS }\end{array}$ & Annually \\
\hline
\end{tabular}




\subsection{QA of tools, devices and procedures}

\subsubsection{Commissioning of geometrical QA tools}

Proper QA can only be done if the pros and cons/limitations of the used QA tools are known. For example, when using geometrical CT or MR compatible markers (dummy markers), the correspondence between these markers and the actual source position should be checked, especially for the first (i.e., most distal) dwell position.

\subsubsection{QA of X-ray, CT or MR markers}

Aim: markers may be used to reconstruct the source path in a catheter and to reconstruct the (first) source dwell position(s). If these markers are used, the following test should be performed.

Test 1: visually inspect for damage or kinks.

Frequency: before use.

Tolerance: should pass.

Test 2: if the marker positions are used for determining source dwell positions, determine locations of the markers with $\mathrm{X}$-ray/CT/MR, and calibrate these against the source dwell positions obtained with autoradiography.

Frequency: annually.

Tolerance: $\pm 0.5 \mathrm{~mm}$.

\section{Note for MR line markers}

Be sure to use geometrically sound RF sequences when visualising such markers with MR imaging.

Additionally, note that partial volume effects can considerably hamper the accuracy of these markers. It is advised to use all available information (e.g. 3D applicator geometry) when reconstructing the (first) dwell position(s) and not to rely on the marker only.

\subsubsection{QA of Source Position Simulator}

Aim: to check the accuracy of the source position simulator (SPS).

Background: to check the first dwell position in flexible tubes, a source position simulator or length gauge tool can be used. The slider with simulator wire may have a limited life expectancy due to heavy or improper use.

Test 1: before clinical use, inspect on damage, kinks, weakening, breaking loose of dummy source capsule, corrosion or general deterioration.

Tolerance: should pass 
Test 2: check consistency with a SPCR. See figure 3.4.

Frequency: annually or on indication.

Tolerance: $\pm 0.5 \mathrm{~mm}$.

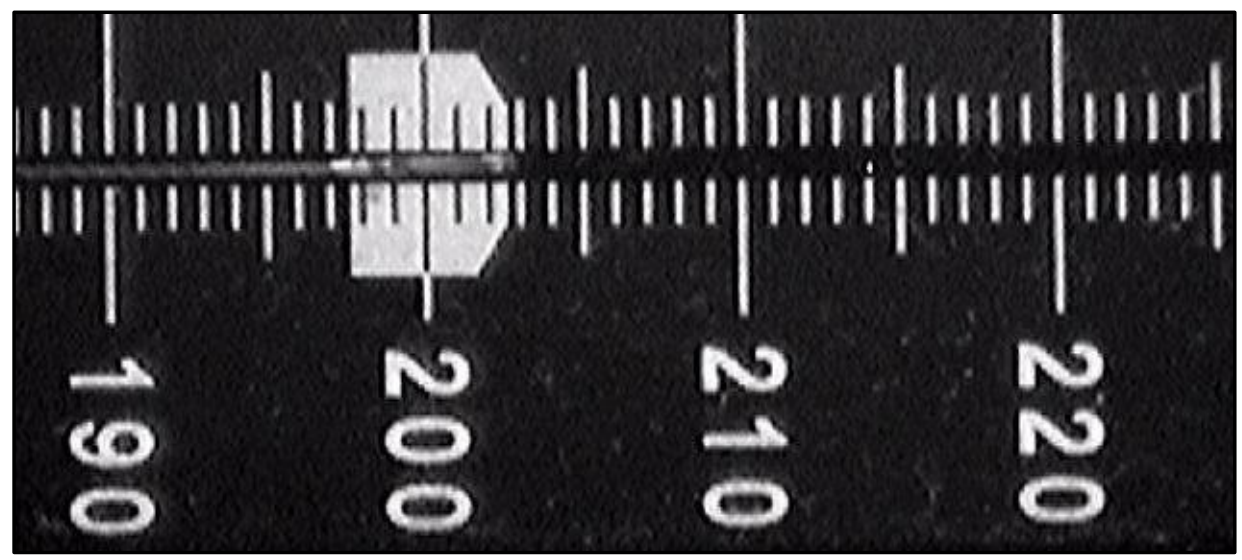

Figure 3.4: Illustration of a source at position 200 in the SPCR.

\subsubsection{QA of Source Position Check Ruler}

Aim: to check the accuracy of the SPCR.

Background: this tool can be used to check the position of the dummy source (visually), the active source

(both visually and by autoradiography) and the Source Position Simulator (SPS). Obviously, the correspondence between these should be checked.

Test: check consistency with suitable phantoms, SPS and/or either dummy or active source.

Frequency: annually.

Tolerance: $\pm 0.5 \mathrm{~mm}$.

\subsubsection{QA of procedures}

\section{Check cable issues/limitations}

In an afterloader system there are two types of cables, one attached to an active source, the other to a dummy source ('check cable'). In some equipment the check cable can also be used for QA purposes. However, the check cable is different from the source cable in several aspects. For example, it is connected to a different drive and the stiffness of the cable is possibly different from the source cable. This poses limitations on the geometrical checks that can be performed with the check cable due to curving and snaking effects (see below).

\section{Curving}

Since the inner diameters of the transfer tubes and applicator are larger than that of the source wire, the trajectory of the source may differ from the centre line. In curved geometries this extra space causes path 
deviations between the intended source position and the actual one. In particular in the case of gynaecological ring-applicators, the trajectory of the source wire along the circular lumen results in a deviation from the expected nominal source path $[20,21,30]$. See figure 3.5 for an illustration. Since drive and stiffness of the check cable are possibly different from the source cable, this effect is not readily measured with the check cable. See also chapter 2.
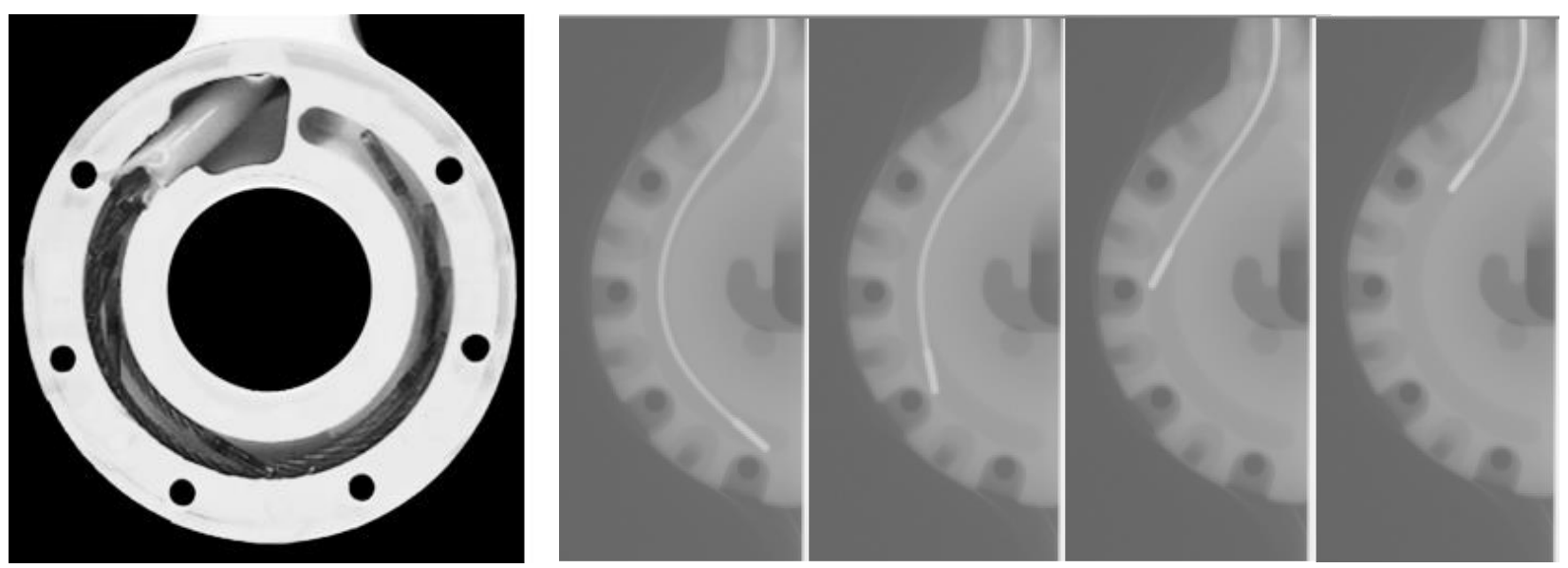

Figure 3.5: Left: from [30]: photograph of a transparent ring phantom, illustrating the real source for a number of dwell positions, depicting the different paths of the source wire. Right: X-ray images of dummy source in interstitial ovoid of the AGA Venezia applicator (Elekta) for different dwell positions.

\section{Snaking}

An additional problem to curving is so-called snaking. Even for straight geometries, the cable may curl in the extra space, resulting in a shorter displacement of the dummy or source than expected. Again, the check cable may not give a proper indication of this problem.

Snaking may also cause a delay in noticing obstructions. The unwinding of the cable may continue without detecting resistance/friction from either source or dummy. This may be especially problematic at the end of the catheter. It is important to evaluate the sensitivity of the system to notice obstruction, for example by steering the dummy to an unattainable position.

\subsection{Chain testing}

To have an estimate on the accuracy of the whole chain of the treatment, it is advised to perform an end-toend test each time a critical element of the treatment chain has been replaced or changed substantially, with potential impact on the dosimetric quality of the treatment. This may include for example a change of imaging device, TPS, applicators, transfer tubes, afterloader. Ideally, in an end-to-end test, the measuring device (i.e., film, TLD, ...), possibly including a phantom, is imaged as a normal patient. On these images a treatment plan is made. This plan is irradiated on the measuring device and compared with the planned 
dose distribution, for instance by exploiting the well-known gamma-agreement index method that is frequently used in external beam radiotherapy. This provides a measure for the accuracy of the whole treatment chain. For external beam radiotherapy, several devices are available to perform such an end-toend test. For brachytherapy, however, such systems are not readily available, and tests often focus primarily on the geometrical aspects of the procedure [31]. The subcommittee accepts this less ideal situation, but wishes to promote advanced testing methods including dosimetrical verifications, as such systems are beginning to appear on the market [32-34].

Performing a complete end-to-end test as described above, including the use of clinical imaging methods, can be a considerable amount of work. A more practical way might be to first establish a solid link between the first source position and an alternative indicator (e.g. tip) and later rely on the verification of that indicator. This can be used to test a sub-part of the chain. This sub-part may be performed on a larger set of transfer tubes and applicators and/or more frequently. It is used to assess the geometrical correspondence between the intended source position and the actual one, thereby verifying the geometrical parameters as used in the TPS for transfer tubes and applicators.

An example of a geometrical chain test is described in section 3.3.5. In principle, we recommend verifying the combined length of the transfer tubes and applicator for all combinations used clinically. For each set, it is advised to use one or more representative specimens.

The first step in this chain test is to link the applicator to a high-resolution (2D or 3D) dose measurement device. For convenience we only consider radiochromic film here. For this test, the absolute dose is less relevant than the geometrical position of the measured dose distribution. Then, predict and mark the location the source should reach on the film, depending on the specific settings in the treatment plan. Next, steer the source to the required dwell position and give an adequate dwell time for a good signal on the film. Finally, determine the centre of irradiation on the film and compare this to the predicted location of the centre of the source. The deviation should be less than $1 \mathrm{~mm}$.

Note that prediction of the expected location is different for different types of applicators. For fixed applicators and rigid needles, the distance between the tip and the first source position might be known. Other techniques may involve high-resolution imaging of the applicator, possibly loaded with dummy markers, and reconstruction of the first source position using the TPS.

For the dosimetric part of the chain test, an audit performed by an external body is valuable. Such an audit has been performed in the United Kingdom [33,34]. Alternatively, a peer-to-peer comparison with other institutes or with alternative dosimetric measurement devices can be done to ascertain dosimetric accuracy. 
The best way to improve brachytherapy locally is an end-to-end audit or peer-to-peer comparison, including imaging, reconstruction, planning and delivery. Examples of such of end-to-end tests are emerging $[35,36]$. 


\section{Radiation safety and security}

HDR and PDR brachytherapy with Ir-192 uses radioactive sources with high activity, up to $500 \mathrm{GBq}$. This imposes demands to both (radiation) safety, and security, preventing radioactive material to be used for criminal or terroristic activities. These demands will be discussed in this chapter. Furthermore, administrative requirements that apply for high-activity sources will be described.

\subsection{Radiation safety aspects related to Ir-192 sources}

Safety aspects which will be considered in this report are the working environment, leakage radiation of the afterloader and the emergency procedure.

\subsubsection{Radiation risk analysis for working with Ir-192 sources}

To achieve an adequate level of a safe working environment with Ir-192 sources it is useful to conduct an ionizing radiation risk analysis. Such an analysis is dictated by Dutch law $[37,38]$. In Belgium it is obliged by the FANC (Federal Agency for Nuclear Control) as a condition of the authorisation for exploitation of such sources, and it will become a legal obligation in the upcoming revision of the ARBIS ('Royal Decreed specifying general conditions on the protection of the population, the employees and the environment against the danger of ionizing radiation'; in Dutch: 'Algemeen Reglement op de Bescherming van de bevolking, van de werknemers en van het leefmilieu tegen het gevaar van loniserende Stralingen') [39]. To conduct such a risk analysis the NCS advisory platform has published a guideline 'RIAS' (Risicoanalyse) [40]. Notable issues from the guideline specific to brachytherapy with Ir-192 sources are:

- a. Proceedings and events

The risk analysis starts with a description of the regular proceedings: which actions are performed where and by whom, e.g. treating patients, performing quality controls, etc. and a description of the 'foreseen unintended events'.

Note: the emergency procedure can be considered, because of the very low probability of occurrence, as a (radiation) incident, rather than a 'foreseen unintended event' and therefore does not need to be considered in the risk analysis [41].

- b. Measures

The risk analysis also contains a description of the measures that already have been taken to minimize radiation exposure or the risk of exposure:

- adequate shielding of the treatment room;

The architectural design of the treatment room has to be such that for persons outside the treatment room a dose constraint of $1 \mathrm{mSv}$ effective dose in one calendar year can be maintained [42], [39]. Keep in mind that personnel working in the control room of the afterloader not only 
receives dose from the attenuated radiation originating from the Ir-192 source but also from other sources of radiation.

- door interlocks;

- warning light indicating "source is out of safe";

- an independent external radiation monitor in the treatment room with a visual and audible indicator for "source is out of safe". The audible indicator is connected to the door interlocks to prevent alarm during regular treatment.

- emergency buttons inside and outside the treatment room;

- warning signs;

Because the treatment room is a controlled area, the entrance of the treatment room must be marked with the warning sign for ionizing radiation (of specified size and colour) and underneath or next to this warning sign two rectangular signs, with black edge, one with the text 'RADIOACTIVE MATERIAL(S)' (Dutch: 'RADIOACTIEVE STOFFEN') and the other with the text 'CONTROLLED AREA' (Dutch: 'GECONTROLEERDE ZONE'): NEN-EN-ISO 7010.

The afterloader itself must also be marked with the warning sign for ionizing radiation and with the rectangular sign with the text 'RADIOACTIVE MATERIAL(S)'.

- wipe test for checking for contamination of source and safe (see also paragraph 2.2.2);

- information and instruction about the risks associated with ionizing radiation; As the Ir-192 sources are high-activity sources all members of the brachytherapy team must be instructed on the requirements for the safe management of the source and the necessary safety provisions. This instruction should be repeated at least every two years [37].

Education and training for preparing and delivery of brachytherapy treatment is subject of chapter 6 . Note: QA of several of these measures is subject of chapter 2. 
A

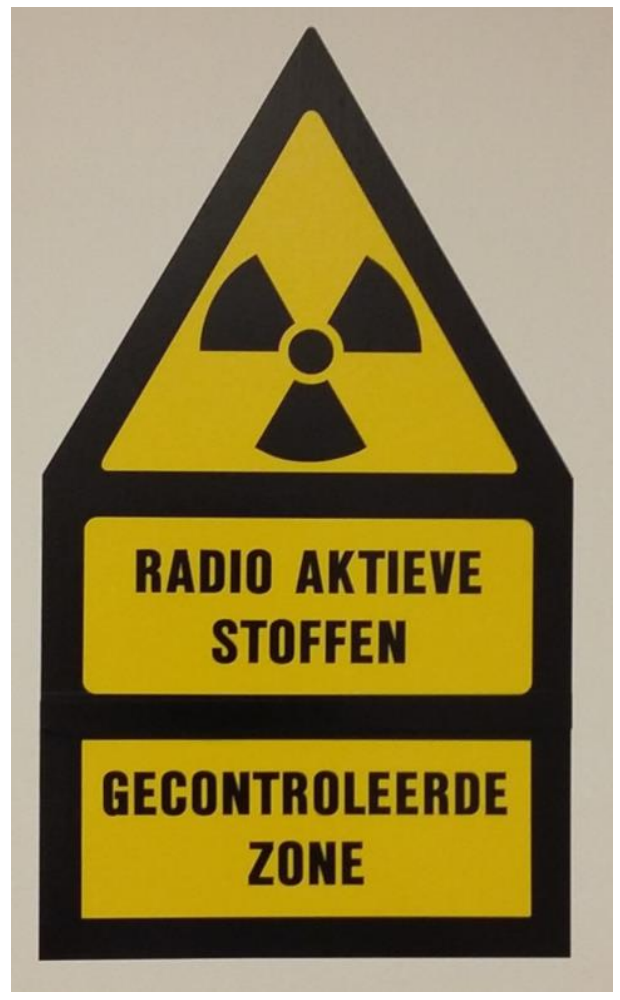

B

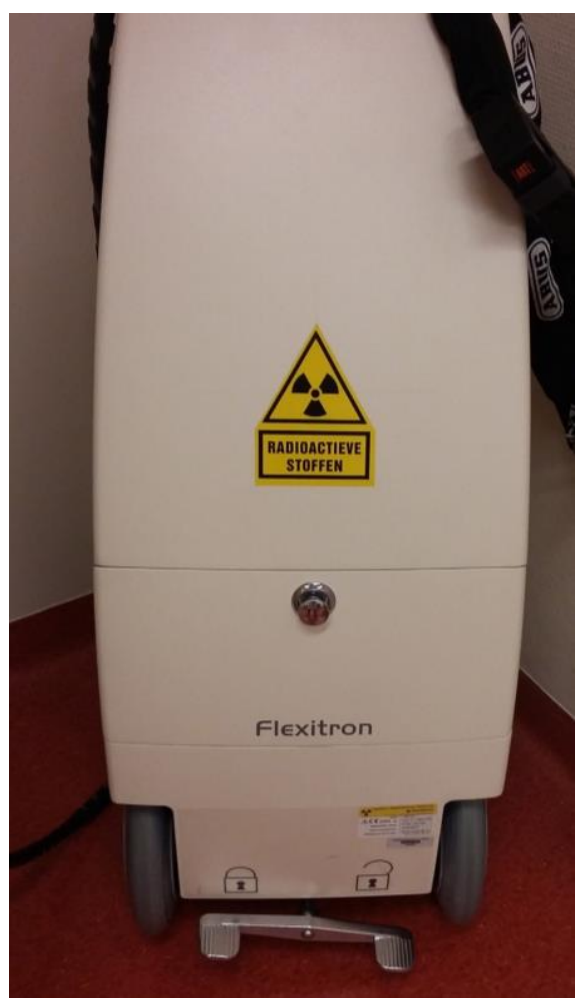

Figure 4.1 A: the warning sign for ionizing radiation and underneath the rectangular signs, with black edge, with the text 'RADIOACTIEVE STOFFEN' and 'GECONTROLEERDE ZONE'

$B$ : the afterloader with the warning sign for ionizing radiation and underneath the rectangular sign with black edge with the text 'RADIOACTIEVE STOFFEN'

\subsubsection{Leakage radiation of the afterloader}

If the Ir-192 source is not used for treatment it will be retracted in the safe of the afterloader (the storage container). The radiation shielding effect of the safe must be such that the leakage radiation stays below a certain level.

For a storage facility or an inherently safe device Dutch law and regulations [42] sets a maximum equivalent dose rate at a distance of $0.1 \mathrm{~m}$ of $1 \mu \mathrm{Sv} / \mathrm{h}$ for leakage radiation.

But the manufacturer of afterloaders designates their afterloader as a source holder for which there is no limit imposed on the maximum leakage radiation by local law. Still, afterloaders have to meet amongst others the European standard IEC 60601 [22], which sets the maximum equivalent dose rate to $100 \mu \mathrm{Sv} / \mathrm{h}$ at $5 \mathrm{~cm}$ and to $10 \mu \mathrm{Sv} / \mathrm{h}$ at $1 \mathrm{~m}$ for storage containers which are used in treatment rooms with restricted access. This restricted access is associated with the radiation safety. In section 4.2 restricted access to the source is an issue from a security point of view. 


\subsubsection{The emergency procedure}

In the exceptional situation that the source fails to retract to the safe in case of malfunctioning of the afterloader or due to breakage of the transport cable, the dose delivered to patient and personnel can be very high $[41,43]$. For this reason there has to be an emergency plan [8]. This emergency plan describes how to handle and which measures have to be taken in case of an emergency. How to handle during an emergency depends on the type of application and the cause of the malfunction. The actions to be taken for different types of applications and different causes of malfunction should be clearly written down. The most important factor in case of an emergency procedure is time. Therefore the procedure has to be trained regularly (at least annually) by all members of the brachytherapy team and the emergency equipment should be available during all treatments.

The emergency instructions and possible error codes of the treatment unit shall be clearly visible in the control room.

The emergency equipment, such as scissors, forceps, emergency container, surgical supplies, cable cutter, hand-held radiation monitor, should be stored in a visible and easily accessible place. This emergency kit has to be checked regularly. Optionally, portable radiation survey meters could be provided to the persons who are assumed to enter the treatment room in case of an emergency.

\subsection{Security aspects related to Ir-192 sources}

Security, as used in this report, means the prevention and detection of, and response to, theft, sabotage, unauthorized access, illegal transfer or other malicious acts involving radioactive materials $[44,45]$.

Due to the increased threat of terrorism, the concern about theft and abuse of radioactive materials has raised. This has led to increased attention for the security of radioactive sources. Recommendations (IAEA Security Series [46] and an EU CBRN ${ }^{3}$ Action Plan [47]) have been drawn up for radioactive materials. In the Netherlands these recommendations have been implemented in Dutch law and regulations [42]. Similar measures are currently being developed in Belgium.

The security measures which have to be taken depend partly on the IAEA category to which the source is assigned. Ir-192 sources used for high/medium dose rate brachytherapy are assigned to category 2 radioactive sources [48].

According to [37] and [42], the following security measures need to be taken to secure radioactive sources: - $\quad$ measures to minimize the risk of theft or misuse.

- $\quad$ supervision of the radioactive source shall be personally or electronically.

\footnotetext{
${ }^{3}$ CBRN: Chemical, Biological, Radiological and Nuclear
} 
- $\quad$ persons who exercise personal supervision shall be authorized for this purpose by the licensee.

- $\quad$ if a radioactive source is not under personal supervision, the security measures shall be such that any attempt of theft or misuse is detected electronically and henceforth measures are in operation resulting in at least a five-minute delay in time a person needs to gain unlawful access to a category 2 source.

- $\quad$ if theft or misuse is detected, this must be notified to the competent authority.

- $\quad$ the licensee must have a security plan including a description of how the source is to be protected.

- $\quad$ only persons for whom it is necessary for the proper exercise of their duties may take note of the security plan. These persons shall submit a certificate of good conduct (Dutch: "Verklaring Omtrent het Gedrag") before taking note of the security plan.

\subsection{Administrative requirements for high-activity sources}

Ir-192 sources, used for brachytherapy, are so called high-activity sealed sources (HASS) because their activity is greater than $8 \times 10^{10} \mathrm{~Bq}$ [37]. In addition to the law and regulations that generally apply to radioactive sources, some additional measures need to be taken for high-activity sources.

\subsubsection{Registration of the Ir-192 source}

Any transferal from a high-activity source to another person must be reported to the competent authority (NL: ANVS). In the Netherlands within two weeks, [37], in Belgium within one working day [39].

Additionally, after the start of each calendar year all high activity sources which are in possession by the undertaking must be reported to the competent authority. In the Netherlands within two weeks [37], in Belgium, within three months [39]).

For reporting the source data of the received source and the disused source to the competent authority standardized forms have to be used. As soon as the activity of a Ir-192 PDR source has dropped down below the activity level of being a high-activity source this has to be reported to the government as if the source is no longer in possession [37,39].

\subsubsection{Financial security with regard to high-activity source}

The undertaking is obliged to safely dispose a disused high-activity source. In the Netherlands, to ensure a safe disposal in case the undertaking is not able to do so, the undertaking must furnish financial security to cover the costs of safe removal of the high-activity source. The minimum amount of the financial security is set by the government [37]. 


\section{Safe Use of Medical Technology in HDR/PDR Brachytherapy}

\subsection{Introduction}

In 2011 the Dutch Hospital Association (NVZ) and the Netherlands Federation of University Medical Centers (NFU) published the agreement 'Safe Use of Medical Technology in the Hospital' (in Dutch: 'Convenant Veilige Toepassing van Medische Technologie in het Ziekenhuis' [18], further referred to as the Convenant), a field standard with the purpose of establishing the framework for the responsibilities regarding the quality assurance of medical technology. The aim of the Convenant is to ensure the safe use of medical technology in the healthcare sector, meaning a safe product in the hands of a trained user in an environment that can guarantee its safe use [18].

This chapter will focus on the recommendations of the Convenant that have a direct bearing on the responsibilities of medical professionals within a brachytherapy team. The general recommendations regarding the responsibilities of the Board of Directors of the hospital/institute, institution procurement rules for medical devices and aids, establishment of a safety management system, etc., fall outside the scope of this chapter. In this report recommendations will be set out for procedures regarding:

- Responsibilities for the use and quality assurance of medical devices used in brachytherapy

- The training and certification of users of medical devices, as well as of technical personnel performing maintenance of these medical devices

- Registration and overview of the maintenance state of medical devices, as well as cleaning/sterilization of reusable medical devices

- Registration of incidents involving medical devices

- Decommissioning of medical devices

Concepts like medical technology, medical devices and aids are often used with overlapping meanings. In this report, we use the term medical technology as defined in the Convenant: medical technology is the application of science and technology for the purpose of enabling a medical treatment. For example, minimally invasive surgery is a medical technology. Medical devices are components of medical technology: any instrument, apparatus or other article used for medical treatment and that is not a medicine is a medical device. The EU Medical Devices Directive [49] states that medical devices are:

Any instrument, apparatus, appliance, software, material or other article, whether used alone or in combination, including the software intended by its manufacturer to be used specifically for diagnostic and/or therapeutic purposes and necessary for its proper application, intended by the manufacturer to be used for human beings." 
"Devices are to be used for the purpose of:

- Diagnosis, prevention, monitoring, treatment or alleviation of disease

- Diagnosis, monitoring, treatment, alleviation of or compensation for an injury or handicap

- Investigation, replacement or modification of the anatomy or of a physiological process

- Control of conception

This includes devices that do not achieve its principal intended action in or on the human body by pharmacological, immunological or metabolic means, but which may be assisted in its function by such means."

To give an example, US-guided transperineal HDR brachytherapy for prostate is a medical technology; the US apparatus, the implant needles, the afterloader and the treatment planning system used are some of the medical devices used within this medical technology.

The safety and reliability of medical technology is determined by measures taken during the whole life cycle of the medical devices that are part of the medical technology. Therefore, in this chapter a distinction will be made (same as in the Convenant) between the introduction phase, the use in clinical practice and the decommissioning phase of medical devices and aids.

\subsection{Responsibilities regarding safe use of medical technology}

Every institution should have procedures wherein responsibilities for the quality assurance/control and use of medical devices are clearly described and assigned to certain specialists within the institution. Responsibilities regarding the safe use of medical technology applied in brachytherapy should be delegated to specific members of the brachytherapy team.

The responsibility for the technical management of medical devices (sources, afterloaders, applicators, TPS, etc.) should be explicitly assigned to a Medical Physics Expert who is part of the brachytherapy team. The Medical Physics Expert brachytherapy is ultimately responsible for:

- Setting up the list of requirements prior to purchasing a medical device, with input from other professionals within and outside the brachytherapy team.

- The acceptance and commissioning of medical devices, as well as their clearance for clinical use (this includes clearance for clinical use after major preventive or corrective maintenance or after major upgrades). This responsibility includes documenting and recording of acceptance and commissioning tests as well as maintenance performed on medical devices.

- $Q A / Q C$ procedures and the maintenance interval of medical devices used in brachytherapy

- Cleaning/sterilization procedures for reusable medical devices as well as for recording cleaning/sterilization cycles that impact the lifetime of a medical device. 
- Decommissioning of medical devices

Some of these responsibilities may be delegated to other specialists. To give some examples, the responsibility for giving clearance for clinical use of brachytherapy equipment after major maintenance could be delegated to medical physics engineers; setting up a procedure for cleaning/sterilization of brachytherapy devices as well as for recording the cleaning/sterilization cycles could be delegated to a RTT.

\subsection{Introduction phase of medical technology and devices}

According to the Convenant, the institution should have a procedure for drafting a plan prior to the implementation of a new medical technology. This plan should address at least the following issues: the necessity of implementation and/or purchase, the consequences for the exploitation, a prospective risk analysis, the necessary training and competences of future users and a periodical evaluation plan. Hereto a purchasing record for every purchase is set up.

In case of custom-made applicators, either manufactured in-house or made to order, a procedure should be put in place for the introduction and use of such applicators in the clinic. This procedure should be based on the EU Medical Devices Directive (European Parliament, 2017). These custom-made applicators can be divided in two categories: applicators made for the sole use of a specific patient or applicators destined for longer term use in the clinic in patients with a particular indication. All custom-made applicators must be manufactured in accordance to written functional requirements of a radiation oncologist or another authorized person. Prior to manufacture a prospective risk analysis should be performed. European manufacturers, either in-house workshop or external company, should be certified per the EN-ISO 13485 quality standard [50]. For each custom-made applicator, a technical file should be set up where all the relevant documents (specifications, test and measurements reports, risk analysis report, etc.) should be kept. Upon manufacturing, the custom-made applicators should pass acceptance and commissioning tests according to local procedures. Applicators custom-made for an individual patient should be accompanied by a manufacturers certificate according to Appendix VIII, article 2.1 of the EU Medical Devices Directive [51]. Custom-made applicators used for the treatment of a particular indication should be accompanied by a manufacturers certificate stating that the applicator meets the essential requirements set out in the Appendix I of the EU Medical Devices Directive [51]. If certain requirements are not met, the reasons for that should be listed in the manufacturers certificate accompanying the applicator.

For in-house developed medical software, a procedure should be put in place covering the following aspects: functional requirements, prospective risk analysis, testing and validation, recording and back-up, versioning and user rights management, in order to prevent software modifications by unauthorized users. 
For leased or loaned equipment, the same requirements as for new equipment should apply. In case of demo equipment, a plan should be drafted encompassing at least the motivation for the placement of the demo equipment, a list of evaluation criteria and a prospective risk analysis.

All medical devices should be registered in a database. The lifetime of these devices should be registered too (expressed in months/years, use cycles, sterilization cycles, etc.) and the user should be triggered when the end-of-life of a certain device is approaching. Specifically, the afterloaders should be labelled in such a way that it is clear to the user if the afterloader can still be used as an HDR, respectively PDR, device for all clinical HDR (or PDR) treatments performed at the specific institution. One example of such a labelling is the placement of a sticker on the afterloader stating the placement date of a new radioactive Ir-192 source. Another example is the placement of green, orange or red boards above the control computer of the afterloader, indicating to the user that the afterloader can be used normally (green), with some limitations stated on the board (orange) or may not be used (red).

Prior to the first clinical use of new brachytherapy devices, users should be adequately trained. A register should be kept of users that are qualified and competent to use a certain medical device.

Furthermore, an emergency procedure should be established and all members of the brachytherapy team should train for emergencies at least once a year. The participation in this training should be documented. Members of the brachytherapy team that have not trained for emergencies in more than one year should not be allowed direct involvement in the treatment of patients.

\subsection{Clinical use phase: medical devices use and maintenance}

The members of the brachytherapy team should be able to check prior to each use whether a certain medical device has clearance for clinical use. Easy access should be provided to a database wherein the maintenance status of medical devices is registered. Also, if the afterloader is in maintenance or otherwise out of order, such status must be clearly visible to the users in order to prevent accidental use in the clinic. Written procedures should be established for the cleaning, sterilization and storage of reusable applicators. Records of the cleaning and sterilization cycles for every applicator should be kept and triggers should be put in place for the applicators whose lifetime is expressed in sterilization cycles in order to enable timely replacement (cf. Chapter 3).

The maintenance standard of leased medical equipment should comply with the institutions own standard. If (part of) the maintenance is performed by an external party/company, those maintenance records should also be kept at the institution.

All incidents regarding medical equipment should be recorded and periodically analysed in order to detect trends or previously unforeseen risk factors. The results of such an analysis can be used to adjust the frequency and/or the scope of periodic corrective and preventive maintenance. Also, a procedure should 
exist for appropriate actions regarding the safety notices sent by the manufacturers of brachytherapy equipment (these safety notices are known as e.g. Field Change Orders (FCO) or Customer Technical Bulletins (CTB)). One member of the brachytherapy team should be designated as single contact person for all manufacturers of brachytherapy equipment for receiving their safety notices.

Members of the brachytherapy team qualified and competent to use a brachytherapy equipment should be registered. This registry should also contain a record of periodical training for every user necessary for maintaining these competences at the required level.

The responsible Medical Physics Expert has to verify that the technical personnel employed by external companies are certified and competent to perform maintenance of medical equipment. For example, in the Netherlands the license of many institutions requires external engineers replacing the Ir-192 source in the afterloader to possess a radiation safety certificate of at least the same level as the institution's technical personnel (see chapter 2). External parties should provide records of necessary certifications prior to deployment of its technical personnel for on-site maintenance.

\subsection{Decommissioning phase of medical devices}

Decommissioned medical devices should be physically removed from the brachytherapy suite, control room or other rooms/spaces where medical devices are used in clinical practice or stored for clinical use. If some decommissioned medical devices are stored elsewhere within the institution, those medical devices should be clearly labelled as being not for clinical use and the inventory records should be modified accordingly.

If a decommissioned medical device is needed for other purposes than patient care, for example educational purposes, and for those reasons it is not physically removed from clinically used spaces, the restricted/limited functionality should be clearly labelled on the respective medical device. 


\section{Organizational aspects of QA in HDR/PDR brachytherapy}

\subsection{Introduction}

HDR/PDR brachytherapy is a radiation treatment modality with a highly multidisciplinary character, not only because of the radiotherapy staff (i.e., radiation oncologist, Medical Physics Expert, RTT, medical physics engineer) involved, but also because the team may extend beyond the RT department to include other clinical specialists from e.g. surgery, radiology, anaesthesiology, and nursery. This requires adequate staffing and coordination of all specialists involved in the safe and accurate preparation and delivery of the treatment. Specific roles, responsibilities, qualifications and tasks of the brachytherapy team members should therefore be clearly defined. The scope and practice of each team member should primarily be based on criteria established by their professional organization and local jurisdiction. Each facility should have written policies and procedures defining the roles and responsibilities of these team members. To this end, it is strongly recommended to define the anticipated or actual flow of the particular HDR/PDR brachytherapy procedures, including all major steps of actions. At each step the team members involved, the critical activities to be performed and the information needed to be captured should be identified. A noncomprehensive overview of the steps of action for the treatment preparation process is given in Table 6.1 by way of example. 
Table 6.1

Example of steps of action for the 'treatment preparation' process for a specific patient.

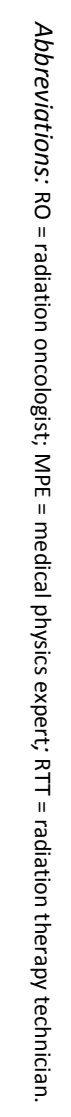
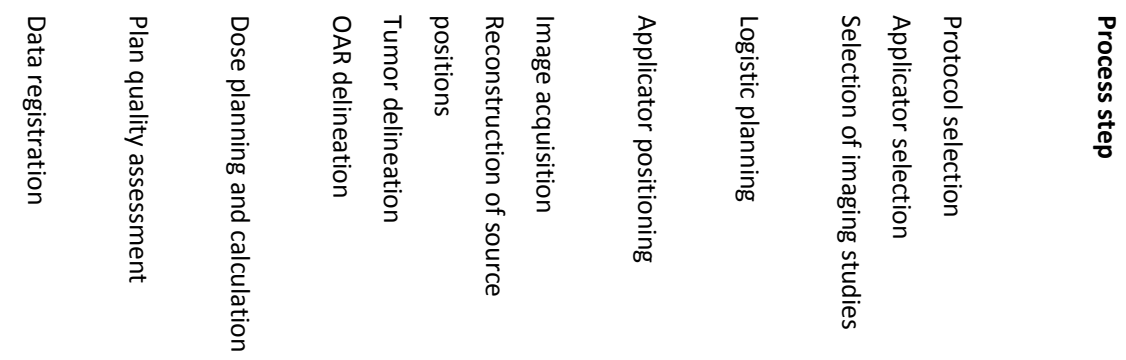

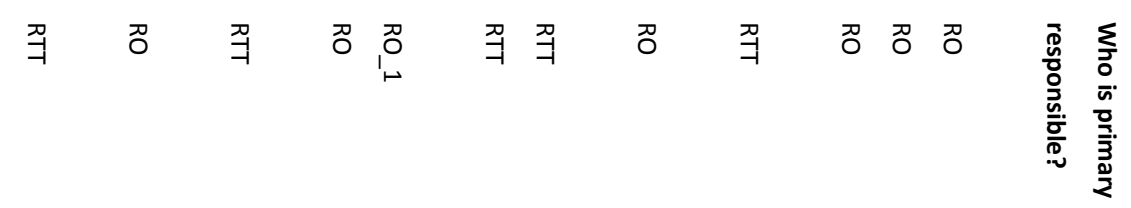

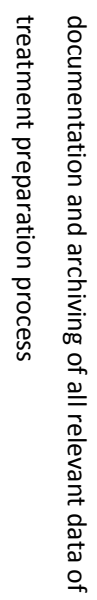
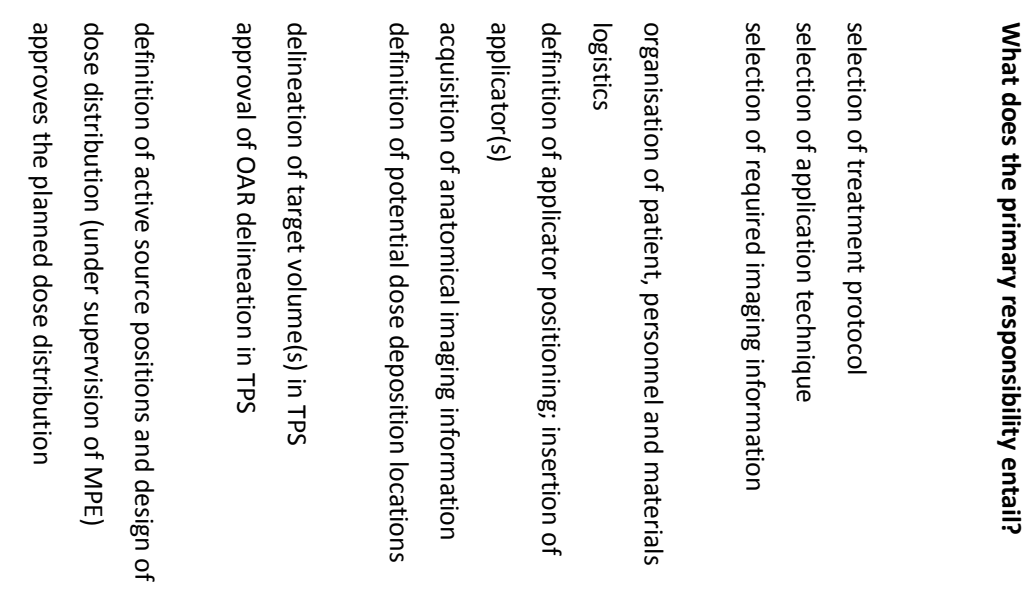

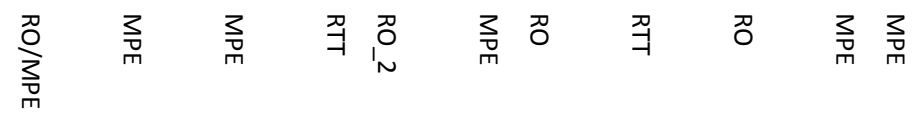
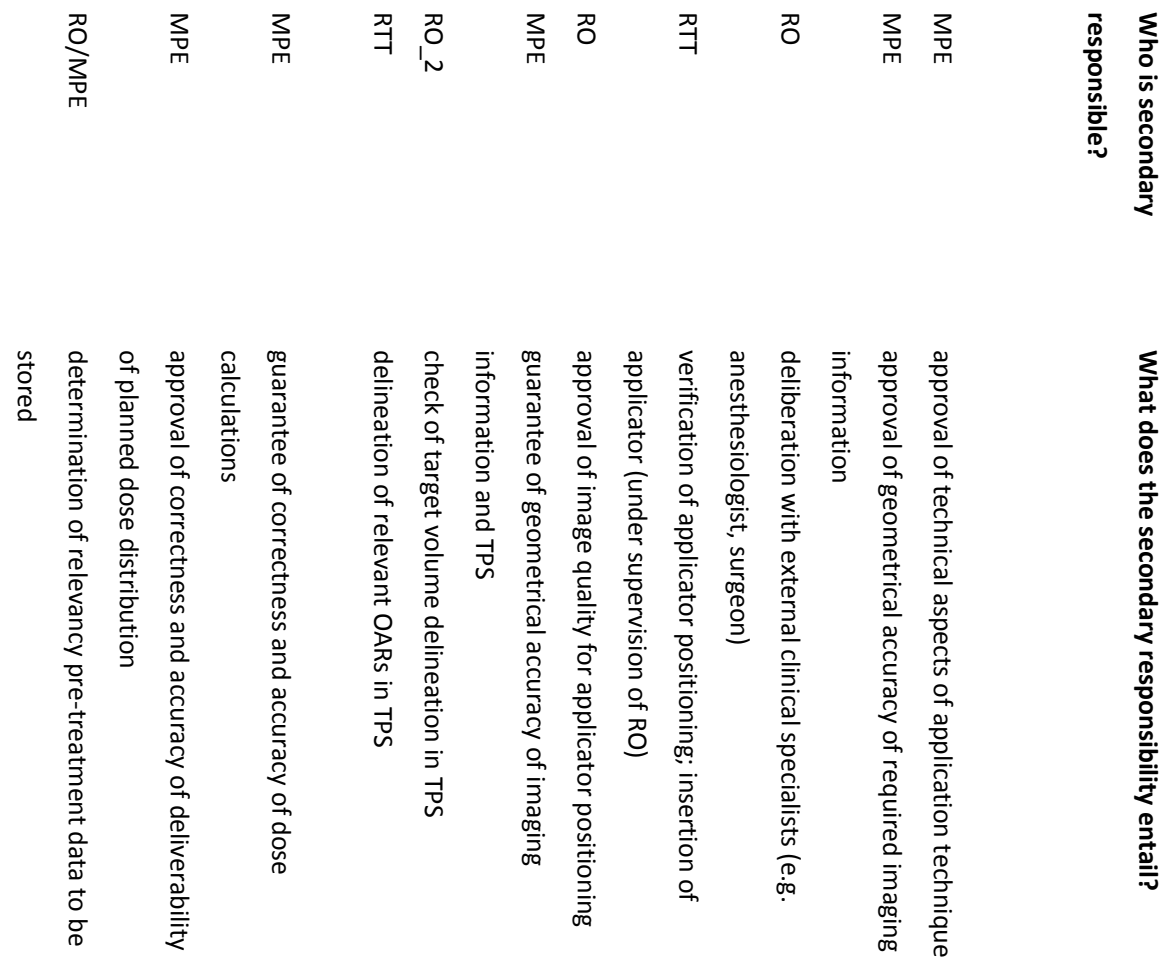


\subsection{The $4 R^{\prime}$ 's of the brachytherapy team}

HDR/PDR brachytherapy is a highly interactive process that requires complex human activities involving cooperation and communication among and beyond the team members. Due to the larger degree of interdepartmental coordination needed compared to external beam radiotherapy, a higher level of cooperation must be achieved.

Sense of duty and individual commitment within the brachytherapy team helps defining the roles and relative responsibilities of the team members. In general, for a successful and safe practice of HDR/PDR brachytherapy, all team members should: be aware of their role, know what their responsibility is (and what not), follow the rules, and have respect for other team members and for the patient. Dutiful attention to details and considerable interaction among the team members is required during crucial steps of the treatment process: applicator inspection and placement, determination of dose specification points or volumes, dose prescription, treatment planning optimization and evaluation, and treatment delivery [11]. All team members should be encouraged to double check each other and identify problems without fear of retribution.

A blame-free culture is a conditio sine qua non for the safe operation of the brachytherapy team. Mutually motivating each other to attain safer treatment by reporting, analysing and learning from errors and nearmisses will stimulate the team bonding and understand each other's skill. This will help build a foundation for trust, which can prevent from miscommunication and conflicts of expectations. The team members should have the ability to contribute to the success of all, and should be able to count on one another. Checklists are recommended as an aid for tracing crucial steps at regular time points prior to or during clinical procedures. The use of checklists has been shown to stimulate the communication between team members, improve their risk awareness and courage to speak up if necessary. Defining a standardized way of communication will help to avoid ambiguities and stay clear of obstinacy and unprofessional behaviour. A briefing and de-briefing of the clinical procedure with all specialists involved in the procedure is desirable to fine-tune expectations, identify potential risks and define actions for improvement of treatment safety and efficacy. 


\subsection{Qualifications of brachytherapy team members}

The process of care in HDR/PDR brachytherapy involves close collaboration between a team of qualified individuals. The primary brachytherapy team consists of, but is not limited to: radiation oncologists, Medical Physics Experts and RTTs. All team members need to be qualified and competent; certification alone is not enough.

All team members have been certified in radiotherapy and possess additional knowledge and expertise to perform HDR/PDR brachytherapy. The radiation oncologist has the workmanship to practise brachytherapy and is competent to carry the ultimate and final medical responsibility, as well as the accountability for all aspects related to patient care. The Medical Physics Expert should be certified in accordance with appropriate national qualifications and is competent to practice independently in the field of brachytherapy medical physics. The medical physics engineer is competent to practice under the supervision of a Medical Physics Expert. The RTT is considered competent if he or she is certified in accordance with the appropriate national qualifications for the designation of RTT and has been sufficiently trained in the field of brachytherapy.

\subsubsection{Specific responsibilities of the team members}

The primary function-specific responsibilities of the brachytherapy team members comprise, but are not limited to [11,52-55]:

Radiation oncologist (RO):

- Development of protocols and new clinical procedures

- Management of clinical aspects of treatment preparation, execution and quality

- Treatment response evaluation

- Patient follow-up

- Patient selection and evaluation

- Protocol selection

- Treatment prescription

- Applicator selection

- Applicator placement (can be delegated to the RTT under strict conditions)

- Request for imaging studies

- Review of imaging for simulation and localization

- Defining target volumes and organs at risk

- Approval of clinical adequacy of treatment plan

- Applicator removal (can be delegated to the RTT under strict conditions) 
Medical Physics Expert:

Note that some of the responsibilities of the Medical Physics Expert may be delegated to medical physics engineers

- Specification of therapy and quality assurance equipment (both hard- and software)

- Radiation safety aspects, in collaboration with the Radiation Protection Expert

- Acceptance testing, commissioning and quality assurance of imaging and therapy equipment, including the treatment planning system

- Quality assurance of treatment planning system

- Management of dose calculation procedures

- Management of technical aspects of treatment planning and treatment delivery

- Innovation, optimization and implementation of treatment techniques

- Establishment and supervision of quality assurance procedures regarding treatment delivery, quality control and regulatory compliance

- Supervision of maintenance of therapy equipment

- Development of new technical procedures

- Emergency procedure training

- Supervision of treatment planning with respect to applicator reconstruction, dose optimization and evaluation

- Treatment plan approval

Radiation Therapy Technologist (RTT):

- Patient data acquisition

- Patient transport, positioning and immobilization

- (Assisting the radiation oncologist during) applicator insertion

- Treatment simulation and localization

- Treatment planning

- Verification of applicators and specific accessories

- Connecting applicators to the afterloader

- Delivering the treatment

- Monitoring the treatment execution from the console

- Applicator removal (under supervision of radiation oncologist)

- Execute and supervise the cleaning, disinfecting, sterilizing and storing of applicators and accessories according to current regulations

- Recording the treatment 


\subsubsection{Specific responsibilities for PDR brachytherapy}

Since the majority of PDR brachytherapy treatment executions is not supervised directly by an attending physician and/or Medical Physics Expert, special precautions must be taken. A dedicated nursing staff should receive training at least once per year on all aspects of radiation safety. This training should include specific responsibilities, identification of all applicators and sources, personal dosimetry procedures, patient handling procedures, emergency procedures, whom to call in case of disruptions, radiation exposure limits, and perspectives on relative risks of radiation exposure [11]. On-call authorized personnel (i.e., physician, Medical Physics Expert or RTT of the brachytherapy team) should be available during treatments. Who performs the functional roles can vary between institutions and should be explicitly stated such that all personnel involved understand the distribution of responsibilities.

\subsubsection{Job description}

It is recommended to have a written description for all roles in the brachytherapy team in the local situation to define the process steps that need to be performed and their associated responsibilities. This information is also valuable for skills management, since it defines the criteria that should be used for assessment and development. Depending on the desired level of detail, a descriptive task list, a list of responsibilities and associated tasks, or a list of specific competences is most useful and manageable (e.g. Table 6.1).

\subsection{Staffing requirements}

Adequate staffing is required for the HDR/PDR brachytherapy team to perform their roles meticulously and safely. Staffing requirement is facility specific and mainly depends on the workload and complexity of the procedures. The recommended brachytherapy team should at least comprise the following personnel:

- 2 radiation oncologists per treatment site having equal responsibilities and the ability to act as mutual stand-in and full sparring partner;

- 2 Medical Physics Experts: one being primarily responsible and one being involved as backup;

- 3 RTT: one being primarily responsible whereas the other(s) have supporting roles.

It is advisable that a medical physics engineer trained in technical aspects of HDR/PDR procedures closely collaborates with the brachytherapy team under the supervision of the Medical Physics Expert.

Understaffing may compromise the treatment quality since the risk of medical errors and professional burnout is expected to increase [43]. Departmental management should be aware that development and maintenance of a safe and efficient HDR/PDR brachytherapy program requires a significant time commitment from all staff involved. Each facility must determine what constitutes an appropriate balance 
between safety procedures, redundancy and staffing levels for routine use in clinical practice. Temporary upscaling of human resources should be budgeted in case of planning a facility, selecting new equipment, acceptance testing and commissioning equipment, developing procedures and staff training.

\subsection{Continuous education and maintenance of certification}

Although there have been some medical events with HDR/PDR brachytherapy, it is generally considered to be a safe treatment modality [43]. Reviewing the errors that lead to recorded medical events has shown that human factors play a dominant role over technical or procedural errors. Events related to human factors resulted from persons involved in the treatment who did not understand the hazards of the procedure or the correct steps, although they had been trained and knew what they were supposed to do, but failed in the execution of this task. Another frequently occurring cause of human factors related events is miscommunication. Continuous education, training and maintenance of both technical and communication skills are therefore mandatory.

Furthermore, it is recommended that all members of the brachytherapy team periodically attend a national or international refresher course in modern brachytherapy techniques to stay informed about contemporary developments in the field. Participation in training and education sessions should be logged for each of the members of the brachytherapy team. It is also recommended to keep a logbook of the HDR/PDR brachytherapy procedures the individual team members have been involved in. Depending on the level of complexity of the procedures, minimum skills needed to perform certain tasks should be defined. It is mandatory to periodically assess staff knowledge and skills, as well as the learning needs of (new) members of the brachytherapy team. An education plan and material as required should be available. It is advised to adapt the scheduling of personnel in accordance with the need for teaching measures as much as possible. It is left to the institutions to practically implement this.

HDR/PDR brachytherapy practitioners should be familiar with guidance documents relevant to any procedure prior to beginning in practice. Recommendations in these documents should be followed and deviations should be supported by clinical studies or pursued in the setting of a clinical trial approved by an institutional review board. Documentation thereof is indispensable. Practitioners need adequate training before beginning in practice. Training should also include a practical component.

The clinical introduction of a new treatment procedure should be accompanied with a careful evaluation of the need for training and proctoring. Once a detailed protocol for the new procedure has been established, a dummy run should be performed with all team members. When major adaptations of the protocol are mandatory, the dummy run should be repeated before the first clinical session. For the introduction of a 
new treatment technique, it is recommended that all team members involved in the new procedure participate in at least 3 proctored cases before performing similar procedures independently.

All members of the brachytherapy team should be trained in the local emergency procedure at least annually. Training participation of each team member should be recorded in a dedicated database.

\subsection{Event reporting}

All adverse events should be gathered in the institutional event report database and analysed structurally to improve the procedures and safety awareness of the team members in order to increase the safety of HDR/PDR brachytherapy. Whenever possible, it is recommended to share these events with colleagues. If equipment is involved, the vendor needs to be informed. In addition, a formal report to the proper authorities should be considered in consultation with the board of directors of the institute.

Furthermore, it is strongly recommended that an electronic or paper log book is kept per therapy machine to allow the RTT to document immediate problems related to machine performance that could affect radiation and mechanical safety.

Manufacturers should at least keep track of all actions, warnings and errors in an automatic log, which is accessible for the user. Preferably all machine parameters are logged and accessible for a service engineer.

\subsection{Internal and external auditing}

An internal audit of the institution's HDR/PDR brachytherapy practice should be conducted. IAEA recommends the Quality Assurance Team for Radiation Oncology (QUATRO) methodology for auditing of radiotherapy practices [56], along with associated detailed medical physics procedures [57]. Both publications contain specific sections on brachytherapy practice auditing. Regular external on-site audits of brachytherapy practice by recognized experts (i.e., peers) is recommended to be part of the institution's quality assurance program. 


\section{References}

[1] Visser AG, Aalbers AH., Burgers J, van der Linden P, Meertens H, van der Steen J, et al. NCS Report 4: Aanbevelingen voor dosimetrie en kwaliteitscontrole van radioactieve bronnen bij brachytherapie. vol. NCS 4. Delft: 1989. doi:10.25030/ncs-04.

[2] Venselaar JLM, Aalbers AHL, Brouwer WFM, Meertens H, Petersen JJ, Schaeken B, et al. NCS Report 7: Recommendations for the Calibration of Iridium-192 High Dose Rate Sources. Delft: 1994. doi:10.25030/ncs-07.

[3] Elfrink RJM, Van Kleffens HJ, Kolkman-Deurloo IKK, Aalbers AHL, Dries WJF, Rijnders A, et al. NCS Report 13: Quality Control in Brachytherapy - Current Practice and Minimum Requirements. Delft: 2000. doi:10.25030/ncs-013.

[4] ASTRO. Safety is No Accident: A Framework for Quality Radiation Oncology and Care. Astro 2012:152.

[5] The Royal College of Radiologists. Towards Safer Radiotherapy. London R Coll Radiol 2008:85. doi:978-1-905034-25-3.

[6] WHO. Radiotherapy risk profile. vol. WHO/IER/PS. Geneve: 2008.

[7] Huq MS, Fraass BA, Dunscombe PB, Gibbons JP, Ibbott GS, Medin PM, et al. Application of risk analysis methods to radiation therapy quality management: Report of AAPM Task Group 100. Med Phys 2016;43:4209-62. doi:10.1118/1.4947547.

[8] Venselaar JLM, Pérez-Calatayud J. A practical guide to quality control of brachytherapy equipment. Eur Guidel Qual Assur Radiother 2004;8.

http://www.estro.org/binaries/content/assets/estro/school/publications/booklet-8---a-practicalguide-to-quality-control-of-brachytherapy-equipment.pdf.

[9] Thomadsen BR, Ph D, Erickson BA, D M, Eifel PJ, Hsu I-C, et al. A Review of Safety, Quality Management, and Practice Guidelines for High-Dose-Rate Brachytherapy. Pract Radiat Oncol 2014;4:65-70. doi:10.1016/j.prro.2013.12.005.

[10] Perez-Calatayud J, Ballester F, Das RK, DeWerd L a., Ibbott GS, Meigooni AS, et al. Dose calculation for photon-emitting brachytherapy sources with average energy higher than $50 \mathrm{keV}$ : Report of the AAPM and ESTRO. Med Phys 2012;39:2904-29. doi:10.1118/1.3703892.

[11] Nath R, Anderson LL, Meli J a, Olch a J, Stitt J a, Williamson JF. Code of practice for brachytherapy physics: report of the AAPM Radiation Therapy Committee Task Group No. 56. American Association of Physicists in Medicine. Med Phys 1997;24:1557-98. doi:10.1118/1.597966.

[12] Aalbers AHL, De Brabandere M, Koedooder C, Moerland MA, Rijnders A, Schaeken B, et al. Dosimetry and quality control of brachytherapy with low-energy photon sources ( 125 I). Delft: 2012. doi:10.25030/ncs-020. 
[13] Kollaard RP, Dries WJF, van Kleffens HJ, Aalbers THL, van der Marel H, Marijnissen HP a, et al. Quality control of sealed beta sources in brachytherapy. vol. 14. Delft: 2004. doi:10.25030/ncs-014.

[14] Nath R, Anderson LL, Luxton G, Weaver KA, Williamson JF, Meigooni AS. Dosimetry of interstitial brachytherapy sources: Recommendations of the AAPM Radiation Therapy Committee Task Group No. 43. Med Phys 1995;22:209-34. doi:10.1118/1.597458.

[15] Rivard MJ, Coursey BM, DeWerd LA, Hanson WF, Huq MS, Ibbott GS, et al. Update of AAPM Task Group No. 43 Report: A revised AAPM protocol for brachytherapy dose calculations. Med Phys 2004;31:633-74. doi:10.1118/1.1646040.

[16] Beaulieu L, Carlsson Tedgren Å, Carrier JF, Davis SD, Mourtada F, Rivard MJ, et al. Report of the Task Group 186 on model-based dose calculation methods in brachytherapy beyond the TG-43 formalism: Current status and recommendations for clinical implementation. Med Phys 2012;39:6208-36. doi:10.1118/1.4747264.

[17] NVZ, NFU, RN. Convenant Veilige Toepassing van medische technologie in het ziekenhuis. 2011.

[18] NVZ, NFU, RN, ZKN. Convenant Veilige toepassing van medische technologie in de medisch specialistische zorg 2016:35.

[19] Khan FM, Gibbons JP. Khan's The Physics of Radiation Therapy. 4th ed. Philadelphia: Wolters Kluwer; 2014. doi:10.1001/jama.1953.02940340098036.

[20] Humer I, Kirisits C, Berger D, Trnkova P, Pötter R, Nesvacil N. Improved source path localisation in ring applicators and the clinical impact for gynecological brachytherapy. J Contemp Brachytherapy 2015;7:239-43. doi:10.5114/jcb.2015.52622.

[21] Awunor OA, Dixon B, Walker C. Direct reconstruction and associated uncertainties of 192Ir source dwell positions in ring applicators using gafchromic film in the treatment planning of HDR brachytherapy cervix patients. Phys Med Biol 2013;58:3207-25. doi:10.1088/0031-9155/58/10/3207.

[22] IEC. Medical electical equipment - Part 2-17: Particular requirements for the basic safety and essential performance of automatically controlled brachytherapy afterloading equipment. 2015.

[23] Van der Laarse R, Niatsetski Y. Flexitron and microSelectron- HDR / PDR afterloaders' transit time and dose. Elekta White Pap 2014.

[24] Varian Medical Systems OS. GammaMed plus TM iX Instructions for Use including the GammaMed plus TM 3 / 24 iX. 2014.

[25] Fonseca GP, Landry G, Reniers B, Hoffmann a, Rubo R a, Antunes PCG, et al. The contribution from transit dose for (192)Ir HDR brachytherapy treatments. Phys Med Biol 2014;59:1831-44. doi:10.1088/0031-9155/59/7/1831.

[26] Bidmead AM, Sander T, Locks SM, Lee CD, Aird EGA, Nutbrown RF, et al. The IPEM code of practice for determination of the reference air kerma rate for HDR 192Ir brachytherapy sources based on the 
NPL air kerma standard. Phys Med Biol 2010;55:3145-59. doi:10.1088/0031-9155/55/11/011.

[27] ICRU. Dose and Volume Specifications for Reporting Intracavitary Therapy in Gynecology. 1985.

[28] ICRU. Dose and Volume Specification for Reporting Interstitial Therapy. 1997.

[29] Hellebust TP, Kirisits C, Berger D, Pérez-Calatayud J, De Brabandere M, De Leeuw A, et al. Applicator reconstruction catalogue by Recommendations from Gynaecological (GYN) GEC-ESTRO working group: Considerations and pitfalls in commissioning and applicator reconstruction in 3D image-based treatment planning of cervix cancer brachytherapy. Radiother Oncol 2010;96:153-60. doi:10.1016/j.radonc.2010.06.004.

[30] Berger D, Dimopoulos J, Pötter R, Kirisits C. Direct reconstruction of the Vienna applicator on MR images. Radiother Oncol 2009;93:347-51. doi:10.1016/j.radonc.2009.07.002.

[31] Roué A, Ferreira IH, Van Dam J, Svensson H, Venselaar JLM. The EQUAL-ESTRO audit on geometric reconstruction techniques in brachytherapy. Radiother Oncol 2006;78:78-83. doi:10.1016/j.radonc.2005.12.004.

[32] Roué A, Venselaar JLM, Ferreira IH, Bridier A, Van Dam J. Development of a TLD mailed system for remote dosimetry audit for 192Ir HDR and PDR sources. Radiother Oncol 2007;83:86-93. doi:10.1016/j.radonc.2007.02.011.

[33] Palmer AL, Lee C, Ratcliffe AJ, Bradley D, Nisbet A. Design and implementation of a film dosimetry audit tool for comparison of planned and delivered dose distributions in high dose rate (HDR) brachytherapy. Phys Med Biol 2013;58:6623-40. doi:10.1088/0031-9155/58/19/6623.

[34] Palmer AL, Bradley DA, Nisbet A. Dosimetric audit in brachytherapy. Br J Radiol 2014;87. doi:10.1259/bjr.20140105.

[35] Awunor OA, Berger D, Kirisits C. A multicenter study to quantify systematic variations and associated uncertainties in source positioning with commonly used HDR afterloaders and ring applicators for the treatment of cervical carcinomas. Med Phys 2015;42:4472-83. doi:10.1118/1.4923173.

[36] Palmer AL, Diez P, Gandon L, Wynn-Jones A, Bownes P, Lee C, et al. A multicentre "end to end" dosimetry audit for cervix HDR brachytherapy treatment. Radiother Oncol 2015;114:264-71. doi:10.1016/j.radonc.2014.12.006.

[37] Overheid NL. Besluit stralingsbescherming (Bs)/Radiation Protection Decree 2018. http://wetten.overheid.nl/BWBR0040509/2018-02-06.

[38] Overheid NL. Regeling stralingsbescherming werknemers 2014/Decree radiation workers 20142018. http://wetten.overheid.nl/BWBR0040573/2018-02-06.

[39] Overheid BE. Koninklijk Besluit 2001. http://www.jurion.fanc.fgov.be/jurdbconsult/consultatieLink?wettekstld=7460\&appLang=nl\&wettekstLang=nl.

[40] NCS. Risico Inventarisatie en Analyse Stralingshygiëne (RIAS)/Risk Inventarisation and Analysis 
Radiation Protection. NCS 2017. http://radiationdosimetry.org/platform/rias.

[41] IAEA. Lessons learned from accidental exposures in radiotherapy. 2000.

[42] Overheid NL. Uitvoeringsregeling stralingsbescherming EZ/Radiation Protection Implementing Regulation (Economic Affairs) 2018. http://wetten.overheid.nl/BWBR0040581/2018-02-06.

[43] ICRP. Prevention of high-dose-rate brachytherapy accidents. Ann ICRP 2004. doi:10.1016/j.icrp.2004.12.002.

[44] IAEA. Code of Conduct on the Safety and Security of Radioactive Sources. IAEA 2004. http://wwwpub.iaea.org/MTCD/publications/PDF/Code-2004_web.pdf.

[45] IAEA. Terminology Used in Nuclear Safety and Radiation Protection. IAEA Saf Gloss 2007:238. http://www-ns.iaea.org/standards/\%5Cnhttp://wwwpub.iaea.org/MTCD/publications/PDF/Pub1290_web.pdf.

[46] IAEA. Security of Radioactive Sources: Implementation Guide. IAEA Nucl Secur Ser No 112009. http://www-pub.iaea.org/MTCD/publications/PDF/Pub1387_web.pdf.

[47] European Commission. Council conclusions on strengthening chemical, biological, radiological and nuclear (CBRN) security in the European Union - an EU CBRN Action Plan - Adoption 2009:1-83. http://register.consilium.europa.eu/doc/srv?l=EN\&f=ST 155052009 REV 1.

[48] IAEA. Categorization of Radioactive Sources. IAEA Saf Stand Ser 2005;No. RS-G-1:70. http://wwwpub.iaea.org/MTCD/publications/PDF/Pub1227_web.pdf.

[49] European Parliament. Council Directive 93/42/EEC. European Union; 1993. doi:2004R0726 - v.7 of 05.06.2013.

[50] CEWAZ Projectgroep. Medische apparatuur zonder CE-markering. 2015.

[51] European Parliament. EU regulation Medical Devices. Off J Eur Union 2017;60. http://eurlex.europa.eu/legal-content/EN/TXT/?uri=OJ:L:2017:117:TOC.

[52] Kubo HD, Glasgow GP, Pethel TD, Thomadsen BR, Williamson JF. High dose-rate brachytherapy treatment delivery: report of the AAPM Radiation Therapy Committee Task Group No. 59. Med Phys 1998;25:375-403. doi:10.1118/1.598232.

[53] IAEA. Setting Up a Radiotherapy Programme: Clinical, Medical Physics, Radiation Protection and Safety Aspects. IAEA 2008.

[54] Fraass B, Doppke K, Hunt M, Kutcher G, Starkschall G, Stern R, et al. American Association of Physicists in Medicine Radiation Therapy Committee Task Group 53: quality assurance for clinical radiotherapy treatment planning. Med Phys 1998;25:1773-829. doi:10.1118/1.598373.

[55] Erickson BA, Demanes DJ, Ibbott GS, Hayes JK, Hsu ICJ, Morris DE, et al. American society for radiation oncology (ASTRO) and american college of radiology (ACR) practice guideline for the performance of high-dose-rate brachytherapy. Int J Radiat Oncol Biol Phys 2011;79:641-9. 
doi:10.1016/j.jijrobp.2010.08.046.

[56] IAEA QUATRO. Comprehensive Audits of Radiotherapy Practices: A Tool for Quality Improvement. IAEA 2007.

[57] IAEA QUATRO. On-site Visits to Radiotherapy Centres: Medical Physics Procedures. IAEA-TECDOC1543A 2007.

[58] Minamisawa RA, Rubo RA, Seraide RM, Rocha JRO, Almeida A. Direct measurement of instantaneous source speed for a HDR brachytherapy unit using an optical fiber based detector. Med Phys 2010;37:5407-11. doi:10.1118/1.3483780.

[59] Wojcicka JB, Yankelevich R, Trichter F, Fontenla DP. Comparison of the transit dose components and source kinematics of three high dose rate afterloading systems. Med Dosim 1999;24:61-5.

[60] Fonseca GP, Viana RSS, Podesta M, Rubo RA, de Sales CP, Reniers B, et al. HDR 192 Ir source speed measurements using a high speed video camera. Med Phys 2015;42:412-5. doi:10.1118/1.4903286.

[61] Fonseca GP, Rubo RA, Minamisawa RA, dos Santos GR, Antunes PCG, Yoriyaz H. Determination of transit dose profile for a (192)Ir HDR source. Med Phys 2013;40:51717. doi:10.1118/1.4802731.

[62] Evans S, Christofides S, Brambilla M. The European Federation of Organisations for Medical Physics. Policy Statement No. 7.1: The roles, responsibilities and status of the medical physicist including the criteria for the staffing levels in a Medical Physics Department approved by EFOMP Counci. Phys Medica 2016;32:533-40. doi:10.1016/j.ejmp.2016.03.001. 
Appendix 1: Safety and physical checks and recommended frequency

\begin{tabular}{|c|c|c|c|c|}
\hline NR & Safety and physical checks & Frequency & Tolerance & Chapter \\
\hline 1 & Source positioning in phantom & $\mathrm{SE} / \mathrm{D}$ & $0.5 \mathrm{~mm} / 1 \mathrm{~mm}$ & Ch2 \\
\hline 2 & Source calibration & SE & $5 \%$ & Ch2 \\
\hline 3 & Timer accuracy & $A$ & $\begin{array}{c}0.1 \text { s or } 1 \%, \\
\text { whichever is greater }\end{array}$ & Ch2 \\
\hline 4 & Room monitor & $4 \mathrm{M} / \mathrm{D}$ & & Ch2 \\
\hline 5 & Warning lights & $4 M / D$ & & Ch2 \\
\hline 6 & Patient camera and microphone & $4 M / D$ & & Ch2 \\
\hline 7 & Interrupt button & $4 \mathrm{M}$ & & Ch2 \\
\hline 8 & Door interlock & $4 \mathrm{M}$ & & Ch2 \\
\hline 9 & Emergency stop & $4 \mathrm{M}$ & & Ch2 \\
\hline 10 & Power loss & $4 \mathrm{M}$ & & Ch2 \\
\hline 11 & Leakage radiation of afterloader & SE & & Ch2 \\
\hline 12 & Contamination test & SE & & Ch2 \\
\hline 13 & Transfer tube attachment lock & $4 M$ & & Ch2 \\
\hline 14 & Transfer tube safety & A & & Ch3 \\
\hline 15 & Transfer tube length & A & $1 \mathrm{~mm}$ & Ch3 \\
\hline 16 & Geometrical check & $A$ & $1 \mathrm{~mm}$ & Ch3 \\
\hline 17 & QA of X-ray, CT or MR markers & $A$ & $0.5 \mathrm{~mm}$ & Ch3 \\
\hline 18 & QA of Source Position Simulator & A & $0.5 \mathrm{~mm}$ & Ch3 \\
\hline 19 & QA of Source Position Check Ruler & A & $0.5 \mathrm{~mm}$ & Ch3 \\
\hline 20 & Emergency equipment & $4 \mathrm{M}$ & & Ch4 \\
\hline 21 & Hand crank functioning & $4 M$ & & Ch4 \\
\hline 22 & Practising emergency procedure & A & & Ch4 \\
\hline
\end{tabular}

$A=$ Annually, $4 M=$ every four months or with source exchange, $S E=$ Source exchange, $D=D a i l y$.

\section{Source positioning in phantom}

Background: Source position is essential for accurate treatment.

Test: $\quad$ For the daily check, only one position should be checked with a transfer tube and from a channel that is used that day. For the check during Source Exchange, more positions should be checked. See also "Length of the treatment tubes"

Frequency: SE/D 
Tolerance: $\quad 0.5 \mathrm{~mm} / 1 \mathrm{~mm}$

\section{Source calibration}

Background: The source is calibrated by the manufacturer and traceable to a national standard laboratory. The RAKR of the source should be determined before taking the source into clinical use. See 2.3

Test: $\quad$ Compare the measured RAKR with the value on the source certificate.

Frequency: SE

Tolerance: $\quad 5 \%$

\section{Timer accuracy}

Background: The accuracy of the timer is crucial for correct dose delivery

Test: $\quad$ Compare the programmed treatment time with the measured treatment time, for both small and large treatment times. This measurement can be combined with the measurement of the transit time, as described in 2.2.1

Frequency: $\mathrm{SE}$

Tolerance: $\quad 0.1$ s or $1 \%$, whichever is greater.

\section{Room monitor}

Background: Radiation in the treatment room is monitored by an independent radiation monitor.

Test: $\quad$ Check that radiation is correctly monitored when radiation is present/absent.

Frequency: $\quad 4 \mathrm{M} / \mathrm{D}$

Tolerance: Pass

\section{Warning lights}

Background: Radiation in the treatment room is indicated by warning lights. The warning lights can be connected to the room monitor or connected to the afterloader and control computer.

Test: $\quad$ Check that warning lights are on/off when radiation is present/absent.

Frequency: $4 \mathrm{M} / \mathrm{D}$

Tolerance: Pass

\section{Patient camera and microphone}

Background: A patient in the treatment room is monitored during treatment with a patient camera and microphone 
Test: $\quad$ Check the proper functioning of both camera and microphone

Frequency: $4 \mathrm{M} / \mathrm{D}$

Tolerance: Pass

\section{Interrupt button}

Background: Pressing the interrupt button brings the source back into the safe

Test: $\quad$ Check the proper functioning by pressing the interrupt button. Check that treatment continues properly after releasing the interrupt.

Frequency: $4 \mathrm{M}$

Tolerance: Pass

\section{Door interlock}

Background: Opening the door during treatment brings the source back into the safe.

Test: $\quad$ Check the proper functioning by opening the door. Check that treatment continues properly after closing the door.

Frequency: $4 \mathrm{M}$

Tolerance: Pass

\section{Emergency stop}

Background: Pressing an emergency stop brings the source back into the safe.

Test: $\quad$ Check the proper functioning by pressing and releasing the various stops. Check that treatment continues properly after releasing the emergency button. Check that a proper report of the partly delivered treatment is generated.

Frequency: $4 \mathrm{M}$

Tolerance: Pass

\section{Power loss}

Background: When power gets lost, the treatment is interrupted immediately. A backup battery provides the power to bring the source back into the safe.

Test: $\quad$ Check the proper functioning by simulating a power loss. Check that treatment continues properly after power has been restored. Check that a proper report of the partly delivered treatment is generated.

Frequency: $4 \mathrm{M}$

Tolerance: Pass 


\section{Leakage radiation of afterloader}

Background: The amount of radiation leakage of the afterloader must comply with international regulations.

Test: $\quad$ Measure the leakage radiation levels at $5 \mathrm{~cm}$ and $100 \mathrm{~cm}$ from the surface of the afterloader.

Frequency: SE

Tolerance: $\quad<100 \mathrm{microSv} / \mathrm{h}$ at $5 \mathrm{~cm} ;<10 \mathrm{microSv}$ at $100 \mathrm{~cm}$ according to IEC 2015

\section{Contamination test}

Background: Source, source cable, dummy and dummy cable should be without radiation contamination. Maximum dose levels are set in national regulations as well as CE-mark of the afterloader.

Test: $\quad$ Swipe-test; often performed by the technician of the source-supplier.

Frequency: SE

Tolerance: Given by national regulations and/or source-supplier.

\section{Transfer tube attachment lock}

Background: Most afterloaders require the transfer tubes to be 'locked' before a treatment can be started. This lock can be a mechanical procedure or the turning of a key.

Test: $\quad$ Check the proper functioning of the lock, by trying to start a treatment with the transfer tubes unlocked and locked.

Frequency: $4 \mathrm{M}$

Tolerance: Pass

\section{Transfer tube safety}

Background: Before the actual source can leave the safe, a check run is performed. If an obstruction is detected the check run is terminated and an error is given.

Test: $\quad$ Check the proper functioning of the dummy run by deliberately creating an obstruction, either by disconnecting the applicator from the transfer tube or by applying a shorter catheter than expected by the system.

Remember that the detection of an obstructed applicator is dependent on the snaking effect: when the dummy or actual source is obstructed the cable will snake slightly inside the applicator and transfer tube.

Frequency: $4 \mathrm{M}$

Tolerance: Pass 


\section{Transfer tube length}

Background: The length of the transfer tube determines the position of the source in the patient. This is the reason the transfer tubes have to be stored straight.

Test: $\quad$ Measure total length of the transfer tube using tape measure on a flat surface or with the tool shown in Chapter 3. Or, measure reference length by performing source position check with Source Position Check Ruler (SPCR), Length Gauge Tool, suitable phantoms, or autoradiography. Compare measured total length with nominal total length and keep record of this measured length.

If transfer tubes are not used for more than 12 months, perform elaborate inspection.

Frequency: A

Tolerance: $1 \mathrm{~mm}$

16 Geometrical check. To ensure that the first (most distal) dwell position, as reconstructed with imaging (X-ray/CT/MR/US), corresponds to the true physical location of the first dwell position.

Background: Depending on the reconstruction method used, the location of the reference point on X$\mathrm{ray} / \mathrm{CT} / \mathrm{MR} / \mathrm{US}$ can be different from the first dwell position. In the TPS, the reconstructed reference point must be coupled to the first dwell position.

Whenever possible, it is advised to check source dwell positions using the radiation of the active source (autoradiography). This can be done for example by film or other high resolution detectors.

Test: $\quad$ Determine the first dwell position and check that it corresponds to the position at commissioning and the value in TPS, using local protocol. Preferably, use the active source with autoradiography, but (3D) X-ray imaging in combination with the dummy source can be allowed, provided that the relation between the position of the dummy source and the active source under clinical conditions (e.g. differences in offset and cable snaking) is known. See also section 3.4 .

Frequency: Annually

Tolerance: $1 \mathrm{~mm}$

\section{QA of X-ray, CT or MR markers}

Background: Markers may be used to reconstruct the source path in a catheter and to reconstruct the (first) source dwell position(s). If these markers are used, the following test should be performed.

Test 1: $\quad$ Visually inspect for damage or kinks.

Frequency: Before use. 
Tolerance: Pass.

Test 2: If the marker positions are used for determining source dwell positions, determine locations of the markers with $\mathrm{X}$-ray/CT/MR, and calibrate these against the source dwell positions obtained with autoradiography.

Frequency: Annually.

Tolerance: $\quad 0.5 \mathrm{~mm}$.

\section{QA of Source Position Simulator}

Background: To check the first dwell position in flexible tubes, a source position simulator or length gauge tool can be used. The slider with simulator wire may have a limited life expectancy due to heavy or improper use.

Test 1: $\quad$ Before clinical use, inspect on damage, kinks, weakening, breaking loose of dummy source capsule, corrosion or general deterioration.

Tolerance: Pass

Test 2: $\quad$ Check consistency with a Source Position Check Ruler. See figure 3.4.

Frequency: Annually or on indication.

Tolerance: $\quad 0.5 \mathrm{~mm}$.

\section{QA of Source Position Check Ruler}

Background: This tool can be used to check the position of the dummy source (visually), the active source (both visually and by autoradiography) and the Source Position Simulator (SPS). Obviously, the correspondence between these should be checked.

Test: $\quad$ Check consistency with suitable phantoms, SPS and/or either dummy or active source.

Frequency: Annually.

Tolerance: $\quad 0.5 \mathrm{~mm}$.

\section{Emergency equipment}

Background: Emergency equipment has to be present in the treatment room or control room. Local protocol describes which equipment is needed, depending on the type of procedures that are performed.

Test: $\quad$ Check if all needed equipment is available and functional.

Frequency: $4 \mathrm{M}$

Tolerance: Pass 


\section{Hand crank functioning}

Background: During an emergency, when the motor of the afterloader cannot return the source to the safe, the hand crank can be used to return the source to the safe.

Test: $\quad$ Check whether the hand crank releases the motor from the source cable and the crank can be turned to return the source to the safe.

Frequency: $4 \mathrm{M}$

Tolerance: Pass

\section{Practising emergency procedure}

Background: During an emergency, when the source cannot return to the safe, immediate action must be taken to remove the source away the patient. Because this procedure is extremely timecritical, practising is necessary on a regular basis.

Test: $\quad$ Re-enact an emergency procedure.

Frequency: Annually for all personnel involved with clinical procedures

Tolerance: Pass 


\section{Appendix 2: Transit dose and dwell time corrections}

The total dose delivered with HDR remote afterloaders can be separated into a dwell component and a transit component resulting from the source movement. For the former, source positioning accuracy and dwell-time accuracy are important uncertainty components for the calculation of the total delivered dose. For the latter, the uncertainty in transit speed of the radiation source is important for accurate dose determination. The transit dose component increases with increasing number of catheters, reducing the total dwell time per catheter or increasing the number of dwell positions with low dwell times. This contribution may become significant ( $>5 \%)$ if it is not corrected for appropriately [25]. Transit dose correction based on average source speed fails to accurately correct the dose, indicating that accurate measurement of the source acceleration and speed profile should be considered [58]. Source kinematics differ between afterloading systems [59].

It cannot be expected from hospitals to perform measurements to determine the instantaneous source speed profiles, as this requires high-speed cameras [60]. In order to be able to determine the transit dose component with high accuracy, accurate measurement of the source acceleration and speed profiles should be incorporated into treatment planning systems or provided by the afterloader manufacturer. Since such data are currently lacking, transit dose should be considered carefully in treatments with high dose rate, several source channels, multiple dwell positions, small dwell times, and multiple fractions [61]. 


\section{Appendix 3: Glossary}

Acceptance

Afterloader

Applicator

Autoradiography

Chain test

Check cable

Control Computer

Control room

Controlled area

Curving

Dose monitor

Dummy source

Dwell position

Dwell time
Acceptance testing of medical devices with the purpose of evaluating the device's compliance with the contractual requirements and specifications and whether it is acceptable for delivery.

A computer controlled remote electromechanical system used for brachytherapy, for placing a sealed source internally or close by the volume to be treated.

A device that can be loaded with a sealed source and is intended to position the source in the location required for treatment.

An image on a radiochromic film produced by the pattern of decay emissions from a distribution of a radioactive substance.

Radioactive sources with activity ratio falling within the limits $1000>A / D>10$ or assigned to this category [IAEA-TECDOC-1344].

Testing a subset of interlinked systems involved in the treatment process.

A second cable without active source (also known as dummy wire/source) in the afterloader, normally used to check the free passage of the transfer tube/applicator assembly.

Computer controlling the afterloader, also known as Treatment Control Station or Control PC.

A separate room outside of the treatment room from where members of the brachytherapy team can remotely administer the treatment and monitor the patient.

An area subject to special rules for the purpose of protection against ionising radiation or preventing the spread of radioactive contamination and to which access is controlled.

The effect that due to the curvature of the bended transfer tube or applicator the actual source-path will deviate from the centre line.

A dose monitoring system incorporating one or multiple radiation detectors used to measure and control the dose level in the treatment room.

An inactive source with the same dimensions as the active source.

Possible position of the source in the applicator at a specified distance from the afterloader.

Time spent by the source at the respective dwell position. 
Emergency storage container

A mobile lead container on wheels used to temporarily store the applicator containing the radioactive source when the afterloader is unable to fully retract the source wire inside the safe.

End-to-end test End-to-end testing is a methodology used to test whether the flow of a treatment process is performing as designed from start to finish.

First dwell position Most distal possible dwell position as seen from the afterloader.

High-activity source A sealed source containing a radionuclide whose activity is equal to or higher than an activity specified in national regulations.

Indexer ring

Length Gauge

Part of the afterloader where the transfer tubes are connected and locked.

A metallic wire of reference length used to check that the channel length of the applicator/transfer tube assembly is within tolerance.

Licensee

See Undertaking.

Markers Radio-opaque or MR contrast markers strung on a wire at fixed positions, used to visualize and check certain reference positions within the source channel, like the end of the channel and/or the first dwell position.

Medical physics expert A medical physics expert is a clinically qualified individual in the field of medical physics who is competent to practise independently in one or more of the specialities of medical physics [62].

Medical physics engineer

In Dutch 'Klinisch Fysisch Medewerker'. An employee who is trained in medical physics and is competent to practise, in close collaboration with the Medical Physics Expert, in one or more of the specialities of medical physics.

Missing applicator Warning given by the Control Computer in case the applicator is not connected properly or at all.

Quality Assurance Set of policies and procedures to maintain the quality of patient care.

Quality Control Set of procedures/tests to validate equipment is operating within tolerance.

Radiation Oncologist Medical specialist that has specific postgraduate training in patient care involving the use of radiation therapy.

Radiation Therapy Technologist

A licensed healthcare professional that plans and administers radiation therapy to patients.

Safe, Storage Container Tungsten casket in which the radioactive source is kept within the afterloader. 
Simulator wire

Snaking

Source

Source Block

Source Step Viewer

Source Position Check Ruler

Step size

Storage facility

Transfer tube

Transit time

Treatment room

Undertaker

Undertaking
Device used for visually checking the source positioning accuracy.

Steel wire (with optional X-ray markers) used in the Source Position Simulator to determine the distal length of an applicator.

The effect that the source cable is curled inside the applicator of transfer tube when the source collides with an obstruction or the end of an applicator.

Radioactive substance within a solid inactive encapsulation.

Mechanism blocking the passage of the radioactive source and wire when a catheter or applicator is disconnected from the transfer tube, or when the transfer tube is disconnected from the afterloader.

See Source Position Check Ruler.

Distance between adjacent dwell positions.

A room used exclusively for storage of radioactive sources that meets specific requirements regarding shielding, access of personnel, fire retardancy, signage and inventory logging (for Dutch requirements see [42]).

Tube used to connect an applicator to the afterloader.

The time the Ir-192 source spends in transit between two dwell positions.

Room used for administering brachytherapy.

Person/persons responsible for the undertaking.

A healthcare provider licensed by the government to use and store radioactive sources or medical devices that emit ionizing radiation. 